\title{
MULTIWAVELENGTH MONITORING OF THE NARROW-LINE SEYFERT 1 GALAXY ARAKELIAN 564. \\ II. ULTRAVIOLET CONTINUUM AND EMISSION-LINE VARIABILITY
}

\author{
S. Collier, ${ }^{1}$ D. M. Crenshaw, ${ }^{2}$ B. M. Peterson, ${ }^{1}$ W. N. Brandt,${ }^{3}$ J. Clavel ${ }^{4}$ R. Edelson,${ }^{5,6}$ I. M. George, ${ }^{7,8}$ \\ K. Horne, ${ }^{9,10}$ G. A. Kriss, ${ }^{11}$ S. Mathur,${ }^{1}$ H. Netzer, ${ }^{12}$ P. T. O’Brien, ${ }^{6}$ R. W. Pogge,${ }^{1}$ \\ K. A. Pounds, ${ }^{6}$ P. Romano, ${ }^{1}$ O. Shemmer, ${ }^{12}$ T. J. Turner,${ }^{7,8}$ and W. Wamsteker ${ }^{4}$ \\ Received 2001 April 30 ; accepted 2001 July 3
}

\begin{abstract}
We present results of an intensive 2 month campaign of approximately daily spectrophotometric monitoring of the narrow-line Seyfert 1 galaxy (NLS1) Ark 564 with the Hubble Space Telescope. The fractional variability amplitude of the continuum variations between 1365 and $3000 \AA$ is $\sim 6 \%$, about a factor of 3 less than that found in typical Seyfert 1 galaxies over a similar period of time. However, large-amplitude, short timescale flaring behavior is evident, with trough-to-peak flux changes of about $18 \%$ in approximately 3 days. We present evidence for wavelength-dependent continuum time delays, with the variations at $3000 \AA$ lagging behind those at $1365 \AA$ by about 1 day. These delays may be interpreted as evidence for a stratified continuum reprocessing region, possibly an accretion disk structure. The $\operatorname{Ly} \alpha \lambda 1216$ emission line exhibits flux variations of about $1 \%$ amplitude. These variations lag those at $1365 \AA$ by $\lesssim 3$ days, and combining this with the line width yields a virial black hole mass limit of $\lesssim 8 \times 10^{6} M_{\odot}$. We caution that the low-amplitude Ly $\alpha \lambda 1216$ variations may indicate the bulk of the emission region is at larger radii. This scenario affects the veracity of our black hole mass upper limit in an uncertain manner owing to the unknown nature of the gas velocity field. Our mass estimate is thus unreliable; however, it is consistent with the independent estimate $M \sim 1 \times 10^{7} M_{\odot}$ of Pounds et al. based on a fluctuation power spectrum analysis of X-ray variability in Ark 564. The black hole mass and $5100 \AA$ luminosity of Ark 564 are consistent with the hypothesis that, relative to Seyfert 1 galaxies, NLS1s have lower black hole masses and higher accretion rates. Other strong emission lines (e.g., C IV $\lambda 1549$ and $\mathrm{He}$ II 21640 ) are constrained to vary with amplitudes of less than $5 \%$. This low level of emission-line variability is different from most Seyfert 1 galaxies, which characteristically display variations of $\sim 10 \%$ on similar timescales.
\end{abstract}

Subject headings: accretion, accretion disks — galaxies: active — galaxies: individual (Arakelian 564) galaxies: nuclei - galaxies: Seyfert

\section{INTRODUCTION}

Narrow-line Seyfert 1 galaxies (NLS1s) were first classified on the basis of their narrow permitted optical emission lines, with $\mathrm{H} \beta$ FWHM $\lesssim 2000 \mathrm{~km} \mathrm{~s}^{-1}$ (Osterbrock \& Pogge 1985). They also exhibit distinctive X-ray properties (e.g., Puchnarewicz et al. 1992; Boller, Brandt, \& Fink 1996; Brandt, Mathur, \& Elvis 1997; Turner et al. 1999; Leighly 1999a, 1999b). These include a steep soft excess with photon

\footnotetext{
${ }^{1}$ Department of Astronomy, Ohio State University, 140 West 18th Avenue, Columbus, OH 43210.

${ }^{2}$ Catholic University of America and Laboratory for Astronomy and Solar Physics, NASA Goddard Space Flight Center, Code 681, Greenbelt, MD 20771.

${ }^{3}$ Department of Astronomy \& Astrophysics, Pennsylvania State University, 525 Davey Laboratory, University Park, PA 16802.

${ }^{4}$ ESA, P. O. Box 50727, 28080 Madrid, Spain.

${ }^{5}$ Department of Physics and Astronomy, University of California, Los Angeles, Los Angeles, CA 90095-1562.

6 Department of Physics and Astronomy, University of Leicester, University Road, Leicester, LE1 7RH, UK.

${ }^{7}$ Joint Center for Astrophysics, Department of Physics, University of Maryland, Baltimore County, 1000 Hilltop Circle, Baltimore, MD 21250.

${ }^{8}$ Laboratory for High Energy Astrophysics, Code 660, NASA/ Goddard Space Flight Center, Greenbelt, MD 20771.

${ }^{9}$ School of Physics and Astronomy, University of St. Andrews, St. Andrews, KY16 9SS, UK.

${ }^{10}$ Department of Astronomy, University of Texas, Austin, TX 78704.

${ }^{11}$ Space Telescope Science Institute, 3700 San Martin Drive, Baltimore, MD 21218.

${ }^{12}$ School of Physics and Astronomy and the Wise Observatory, The Raymond and Beverly Sackler Faculty of Exact Sciences, Tel Aviv University, Tel Aviv 69978, Israel.
}

index $\Gamma_{\text {soft }}>3$ (photon flux $P_{E} \propto E^{-\Gamma}$ ), a steep hard power law with $\Gamma_{\text {hard }}>2.5$, and rapid, short timescale variations (e.g., $\sim 30 \%$ in 1500 s). Their UV/optical properties place them at one extreme of the Boroson \& Green (1992) primary eigenvector that has been identified in a principal component analysis; specifically, NLS1 classification correlates with strong optical $\mathrm{Fe}$ II and weak [O III] $\lambda 5007$ emission. NLS1s are thus potentially useful in identifying the underlying physics that defines the primary eigenvector.

A number of scenarios have been posited to explain NLS1 properties:

1. NLS1s may have larger broad-line region (BLR) sizes compared to Seyfert 1 galaxies (S1s). The steeper soft excesses imply higher ionizing fluxes than S1s with comparable luminosities. Under certain conditions this may lead to larger BLR sizes and, hence, smaller permitted line widths, assuming they reflect virialized motions about the putative black hole (Guilbert, Fabian, \& McCray 1983; Wandel \& Boller 1998).

2. NLS1s may be low inclination $i$, nearly face-on systems (Osterbrock \& Pogge 1985). The line widths, attributable to orbital, virialized motions in a common plane, are decreased by $\sin i$, and the strong soft X-ray fluxes are reconcilable with accretion disk models (e.g., Madau 1988). However, this orientation scenario has problems explaining the low [O III] luminosities often observed (e.g., Boroson \& Green 1992).

3. NLS1s may have relatively low black hole masses with higher than normal accretion rates, compared to S1s 
(Pounds, Done, \& Osborne 1995). The smaller line widths are attributable to the reduced gravitational potential in which they form, and higher accretion rates result in luminosities that are large for their masses.

Balmer $(\mathrm{H} \beta)$ BLR sizes and masses have been measured for about 40 active galactic nuclei (AGNs), including five NLS1s (Wandel, Peterson, \& Malkan 1999; Kaspi et al. 2000; Peterson et al. 2000). These results suggest that NLS1s have BLR sizes comparable to those of S1s with similar optical luminosities. Moreover, NLS1s and S1s delineate a broad mass-luminosity relationship with the former sources populating the low-mass extremum of this relationship. This is consistent with the hypothesis that NLS1s are undermassive black hole systems with higher accretion rates and/or sources viewed nearly face-on.

To distinguish between various models requires systematic measurements of the black hole masses $M$, mass accretion rates $\dot{M}$, BLR sizes $R_{\mathrm{BLR}}$, and source inclinations $i$ of NLS1s and other S1s. These key parameters may all be measured directly or inferred through application of echomapping (reverberation) techniques (Blandford \& McKee 1982; Peterson 2001) that use the relative responses of continuum and emission-line components to strongly constrain the nature of the responding emission regions. The responsivity-weighted BLR size is given approximately by $R_{\mathrm{BLR}}=\tau c$, with $\tau$ the time lag, measured from crosscorrelation analysis, between the continuum and delayed, radiatively driven emission-line variations. Combining $R_{\text {BLR }}$ with the emission-line velocity FWHM $V_{\mathrm{FWHM}}$, assumed to be gravitationally determined and derived from the variable line profile, virial masses $M$ follow from $M=$ $f V_{\mathrm{FWHM}}^{2} R_{\mathrm{BLR}} / G$, with $f$ a factor of order unity that depends on the detailed emission-line gas distribution (and which may be determined by measuring the velocity-dependent emission-line response). The results of Wandel et al. (1999), Kaspi et al. (2000), and Peterson et al. (2000) are based on these reverberation techniques. The magnitude and wavelength dependence of any continuum time delays, when combined with the spectral energy distribution, may be used to constrain the product $M \dot{M}$ under certain conditions (Collier et al. 1999); hence, $\dot{M}$ follows given $M$ from the associated emission-line reverberation measurement. The width of the continuum delay distribution at many wavelengths and the velocity dependent emission-line response (e.g., Welsh \& Horne 1991) may be used to measure the source inclination. These methods for measuring $\dot{M}$ and $i$ have not been applied with much success to existing data sets, since they lack sufficient signal-to-noise ratio and monitoring duration. Other methods for measuring $i$ include accretion disk fitting to $\mathrm{Fe} \mathrm{K} \alpha$ (e.g., Nandra et al. 1997) and combined UV continuum and $\mathrm{H} \beta$ measurements (Rokaki \& Boisson 1999).

We undertook a program of coordinated multiwavelength observations of the NLS1 Ark $564(z=0.0247$; Huchra, Vogeley, \& Geller 1999) in the summer of 2000. Ark 564 is the brightest known NLS1 in the $2-10 \mathrm{keV}$ band, with hard X-ray luminosity $L_{\mathrm{X}} \simeq 10^{43.4} \mathrm{ergs} \mathrm{s}^{-1}$ (Turner et al. 2001, hereafter Paper I). It has a steep power-law continuum $\left(\Gamma_{\text {hard }} \approx 2.5\right)$, ionized $\mathrm{Fe} \mathrm{K}$ line features, and a steep soft excess, as described in Paper I. A 35 day $A S C A$ observation shows evidence for flux variations of $\approx 36 \%$ (Paper I; Edelson et al. 2001). Optical monitoring by Giannuzzo et al. (1998) reveals $\mathrm{H} \beta$ variations of about $8 \%$ on timescales of about $4 \mathrm{yr}$, rather lower than observed in other S1s over similar periods (e.g., about $25 \%$ for NGC 5548). Optical spectra reveal the presence of strong [Ca II] and $\mathrm{Fe}$ II emission (van Groningen 1993). No program of UV monitoring has been previously undertaken on this source, but $\S 3$ presents an archival IUE observation. Our program included $A S C A$ (Paper I), HST (this paper), optical (Shemmer et al. 2001, hereafter Paper III), and Rossi X-Ray Timing Explorer (Pounds et al. 2001) observations and represents the most comprehensive contemporaneous multiwavelength study of an NLS1 to date. In this paper, we present the UV continuum and emission-line variability results obtained with $H S T$ during the period 2000 May 9-July 8 . These observations were primarily intended to measure the effective size of the UV BLR of an NLS and permit measurement of a virial mass $M$ based on multiple emission lines, thereby further constraining the nature of NLS1s. Ark 564 also has a rich UV absorption spectrum (Crenshaw et al. 1999); discussion of our absorption-line results will be deferred to a later paper. In $\S 2$, we describe the observations. We discuss the properties of the mean spectra in $\S 3$. The continuum and emission-line variability are presented in $\S \S 4$ and 5, respectively. Our results are discussed and summarized in $\S 6$ and 7, respectively.

\section{OBSERVATIONS}

We observed the nucleus of Ark 564 with the Space Telescope Imaging Spectrograph (STIS) on the Hubble Space Telescope (HST) on 46 occasions during the period 2000 May 9-July 8. The first five visits were separated by intervals of $\sim 5$ days. Beginning on the fifth visit (2000 May 29), the sampling interval was decreased to $\sim 1$ day for the remaining monitoring period. We obtained low-resolution spectra during each visit with the G140L and G230L gratings, thereby providing full UV coverage at a spectral resolution of approximately $1.2 \AA$ over the range $1150-1730$ $\AA$ and $3.2 \AA$ over the range $1570-3150 \AA$. We used the $52^{\prime \prime} \times 0$ ".5 slit for the low-resolution spectra to maximize throughput and ensure accurate absolute photometry. As Ark 564 is a point source in the STIS spectral images, there was no significant resolution degradation by using the wide slit. The exposure times were 1200 and $520 \mathrm{~s}$ for each G140L and G230L spectrum, respectively, except on 2000 May 29 when the respective exposure times were 1434 and $816 \mathrm{~s}$. To estimate the effects of the intrinsic absorption on the UV emission-line profiles, we obtained high-resolution echelle spectra of the nucleus on 2000 May 29 with the E140M grating at a spectral resolving power of $\lambda /$ $\Delta \lambda \approx 45,000$. The E140M spectra were obtained through the $0.2 \times 0$ ". 2 aperture during four consecutive orbits to yield a combined exposure time of 10,310 s. The spectra were reduced using the IDL software developed at NASA's Goddard Space Flight Center for the STIS Instrument Definition Team (Lindler 1998). For the low-dispersion spectra, we used standard point-source processing with an extraction height (perpendicular to the dispersion) of 0 "'275 to obtain flux-calibrated G140L and G230L spectra as a function of wavelength.

Small wavelength calibration uncertainties exist between spectra of a given grating. To remove these effects, relative wavelength calibration of spectra for each grating is required. The methodology we employ is analogous to that described by Korista et al. (1995, except we do not subtract a continuum fit from each spectrum). We cross-correlate the 
spectra near the emission-line peaks of Ly $\alpha \lambda 1216$, C IV $\lambda 1549$, and He II $\lambda 1640$ for the G140L grating, and near the peaks of C III] $\lambda 1909$ and $\mathrm{Mg}$ II $\lambda 2798$ for the G230L grating. Hence, we determine the nearest whole pixel shifts for each spectrum relative to its appropriate mean spectrum. The majority of the spectra (about 90\%) required either a 1 or 0 pixel shift, and the remaining spectra required a 2 pixel shift. The uncertainty in our relative wavelength calibration is on the order of \pm 0.5 pixels, i.e., about $0.6 \AA$ and $1.7 \AA$ for the G140L and G230L gratings, respectively. We made no attempt to intercalibrate the G140L and G230L spectra, given their difference in resolution.

\section{MEAN SPECTRA}

The observed mean and rms of the $46 \mathrm{G} 140 \mathrm{~L}$ and G230L spectra are presented in Figures 1 and 2, respectively. The rms spectra have been corrected for the bias owing to measurement errors. Furthermore, these spectra have been corrected for Galactic reddening using $E(B-V)=0.06$ mag. (Schlegel, Finkbeiner, \& Davis 1998). We note narrow-line Balmer decrement and $\mathrm{He}$ II $\lambda 1640 / \mathrm{He}$ II $\lambda 4686$ ratios suggest that internal reddening is potentially large for Ark 564 (Walter \& Fink 1993; Paper III), i.e., $E(B-V) \approx 0.2$ mag. Determination of the internal reddening in Ark 564 will be discussed in a forthcoming paper by Crenshaw et al. (2001). The mean spectra are rich in both emission and absorption features. The prominent emission features have been identified and labeled in Figures 1 and 2. Many of the unlabeled, poorer contrast features may be associated with $\mathrm{Fe}$ II emission. Intrinsic UV absorption in Ly $\alpha$ $\lambda 1216, \mathrm{~N} \mathrm{v}$ $\lambda 1240$, Si IV $\lambda 1397$, and C IV $\lambda 1549$ is present, along with Galactic absorption in lines such as Si IV $\lambda 1397$ and $\mathrm{Mg}$ II $\lambda 2798$ (Crenshaw et al. 1999). We have made no attempt to identify the complex of absorption features shortward of Ly $\alpha \lambda 1216$, since this spectral region is affected by timedependent residuals owing to imperfect subtraction of geocoronal Ly $\alpha$ emission. We defer discussion of the rms spectra to $\S 5$.

In order to quantify the prominent emission-line characteristics, we determine pure emission-line spectra from which appropriate mean spectral measurements are then made. This was done through cubic spline interpolations over the absorption features and subtraction of a power-law fit to the continuum. Our spectral measurements (to be discussed in this section) assume zero internal reddening. The continuum is defined by four nominally line-free bands chosen by visual inspection of the mean spectrum (Fig. 1): $1155-1180 \AA$, $1350-1380 \AA, 1460-1500 \AA$, and $1620-1660 \AA$. These bands only approximate the true continuum level, since Balmer recombination continuum, Fe II blends, and other weak broad emission contaminate them to some extent. The rms spectrum (Fig. 1) indicates these bands are not notably biased by line variability. The best-fit continuum is described by $F_{\lambda}=k(\lambda / 1000)^{\alpha}$ with $\alpha=-0.88 \pm 0.01$ and $k=(1.56 \pm 0.01) \times 10^{-14} \mathrm{ergs} \mathrm{s}^{-1} \mathrm{~cm}^{-2} \AA-1$. The continuum fit uncertainties are purely statistical and should

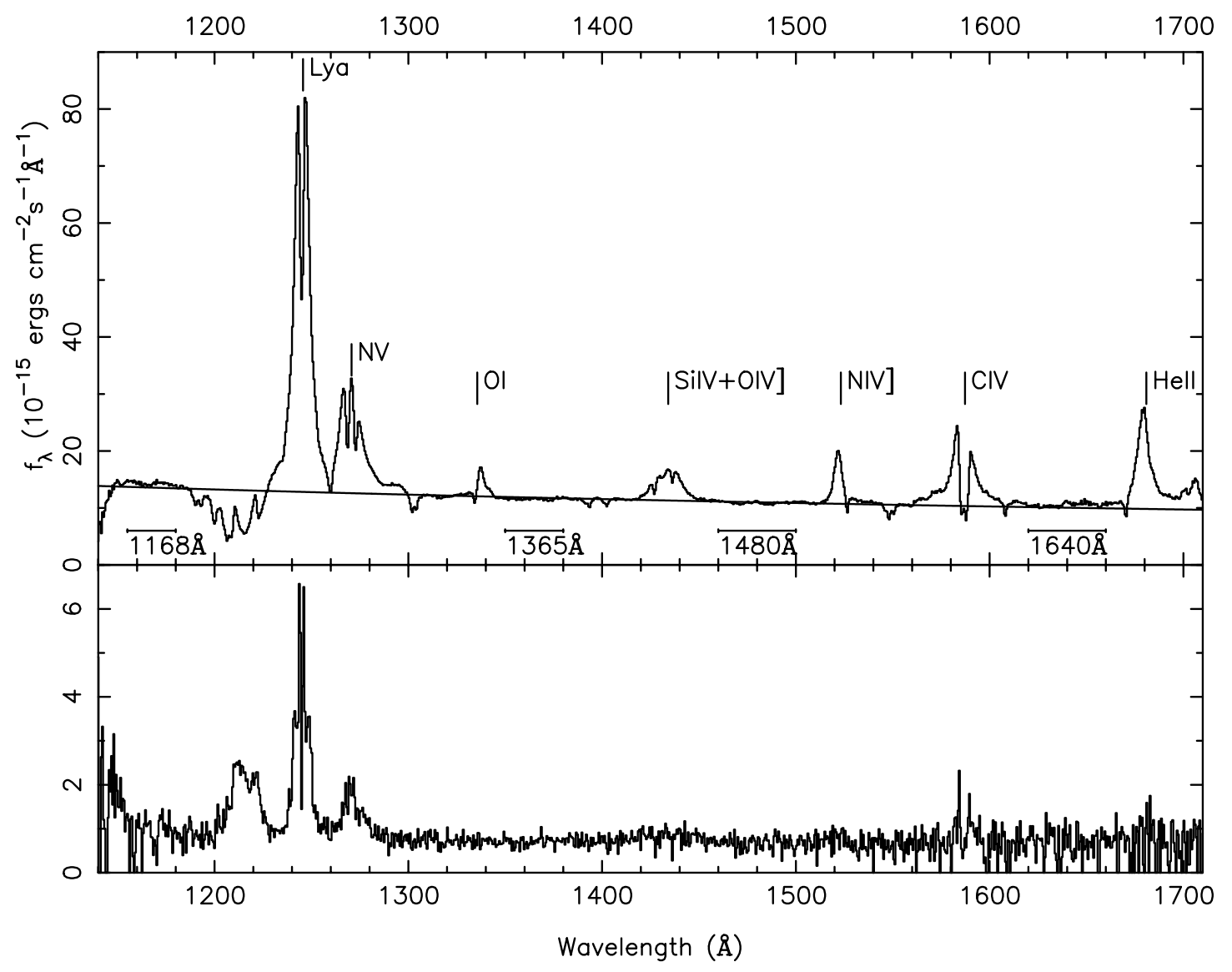

FIG. 1.- Observed mean (top panel) and rms (bottom panel) spectra of the 46 G140L spectra. These spectra have been corrected for Galactic reddening using $E(B-V)=0.06 \mathrm{mag}$. The mean spectrum displays many emission and absorption-line features; the solid line denotes the best-fit power-law continuum (see text for further details). The continuum bands used in this paper are as indicated. The rms spectrum shows evidence for Ly $\alpha \lambda 1216, \mathrm{~N} v \lambda 1240, \mathrm{Si}$ IV + O IV] $\lambda 1400, \mathrm{C}$ IV $\lambda 1549$, and He II $\lambda 1640$ variations with amplitudes of less than $4 \%, 5 \%, 6 \%, 5 \%$, and $4 \%$, respectively. 


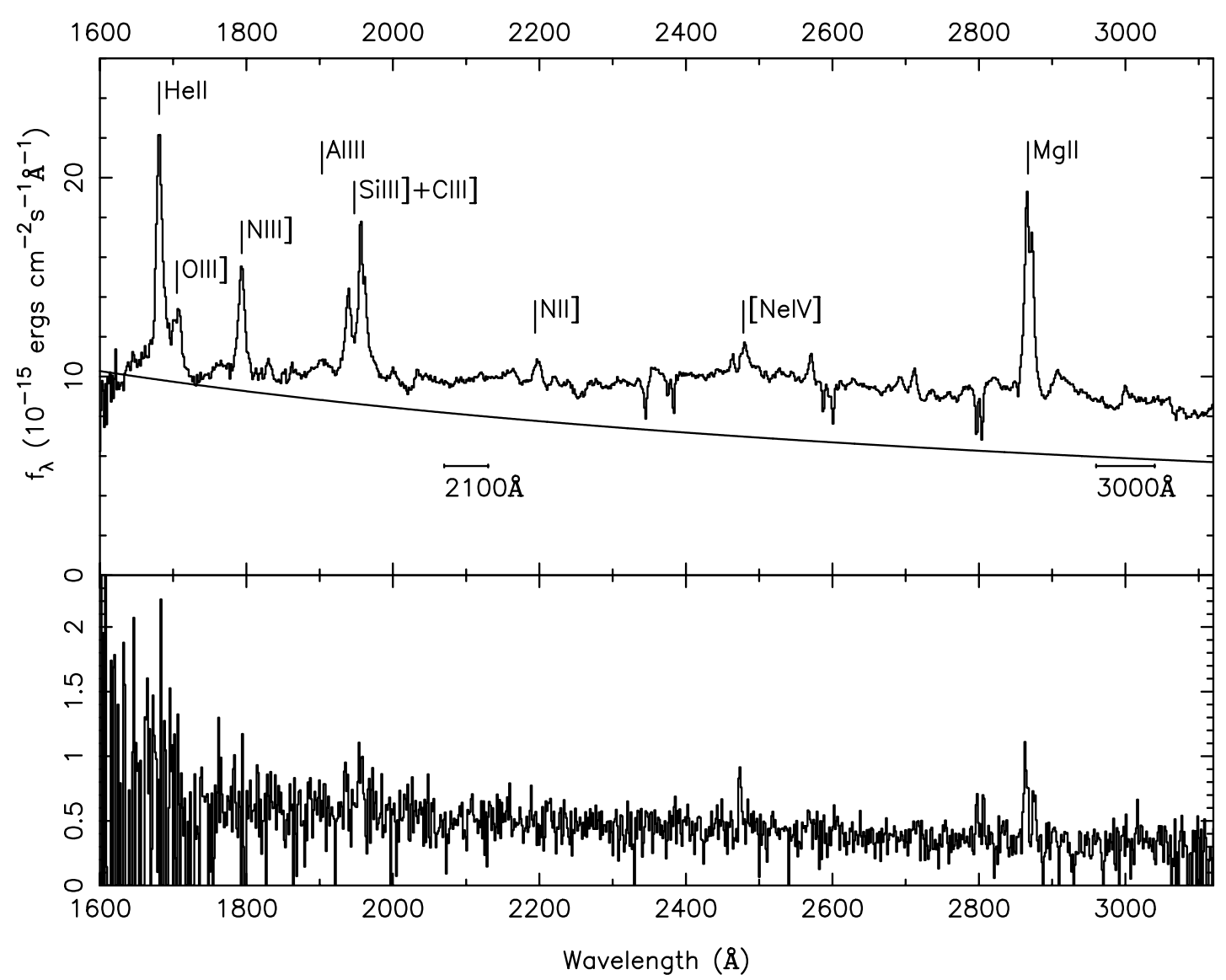

FIG. 2.-Observed mean (top panel) and rms (bottom panel) spectra of the $46 \mathrm{G} 230 \mathrm{~L}$ spectra. These spectra have been corrected for Galactic reddening using $E(B-V)=0.06 \mathrm{mag}$. The mean spectrum displays many emission and absorption-line features. The prominent emission ines are marked, including the continuum bands utilized in $\S 4.1$. The rms spectrum shows evidence for Si III $\lambda 1892, \mathrm{C} \mathrm{III}] \lambda 1909$, and $\mathrm{Mg}$ II $\lambda 2798$ variations with amplitudes of less than $6 \%$, $6 \%$, and $4 \%$, respectively.

be treated with caution, since systematic errors owing to reddening, for example, are nonnegligible. This UV continuum is redder than $F_{\lambda} \propto \lambda^{-1.34}$, typically observed in quasars (O'Brien, Wilson, \& Gondhalekar 1988). This may be due to, for example, disk irradiation (assuming the UV emission arises in an accretion disk) and/or internal reddening. We choose to extrapolate this continuum into the G230L spectral range, since here the continuum level is poorly defined, as the aforementioned contaminants strengthen. We note, in this regime, the extrapolated continuum is about $30 \%$ below the local pseudocontinuum defined largely by the Fe II blends. The validity of the above continuum fit awaits detailed consideration of these $\mathrm{Fe}$ II blends and corrections for internal reddening.

Our emission-line spectral measurements are detailed in Table 1. For each emission line in column (1) we give the

TABLE 1

EMISSION-LINE CHARACTERISTICS

\begin{tabular}{|c|c|c|c|c|c|}
\hline $\begin{array}{l}\text { Line } \\
\text { (1) }\end{array}$ & $\begin{array}{c}\mathrm{FW}_{0.5} \\
\left(\mathrm{~km} \mathrm{~s}^{-1}\right) \\
(2)\end{array}$ & $\underset{\left(\times 10^{13} \underset{0.5}{\left.\mathrm{ergs} \mathrm{s}^{-1} \mathrm{~cm}^{-2}\right)}\right.}{(3)}$ & $\begin{array}{c}\mathrm{FW}_{0.2} \\
\left(\mathrm{~km} \mathrm{~s}^{-1}\right) \\
(4)\end{array}$ & $\underset{\left(\times 10^{13} \mathrm{~F}_{0.2}^{\mathrm{ergs} \mathrm{s}^{-1} \mathrm{~cm}^{-2}}\right)}{(5)}$ & $\begin{array}{l}\lambda_{0.2}^{\mathrm{cen}} \\
(\AA) \\
(6)\end{array}$ \\
\hline $\operatorname{Ly} \alpha \ldots \ldots \ldots \ldots \ldots$ & 2114 & 5.30 & 3553 & 6.51 & 1245.2 \\
\hline $\mathrm{N} v \ldots \ldots \ldots \ldots \ldots$ & 2809 & 1.98 & 4725 & 2.56 & 1270.5 \\
\hline$\left[\mathrm{O}_{\mathrm{I}}\right] \ldots \ldots \ldots \ldots \ldots$ & 788 & 0.15 & 1563 & 0.20 & 1338.1 \\
\hline $\mathrm{Si}$ IV $+\mathrm{O}$ IV $] \ldots \ldots$ & 3270 & 0.70 & 4859 & 0.84 & 1433.9 \\
\hline $\mathrm{N}$ iv $] \ldots \ldots \ldots \ldots$ & 928 & 0.35 & 1487 & 0.44 & 1521.6 \\
\hline $\mathrm{C} \operatorname{iv} \ldots \ldots \ldots \ldots \ldots$ & 1934 & 1.33 & 3469 & 1.76 & 1586.3 \\
\hline He II .............. & 1195 & 0.91 & 2585 & 1.38 & 1679.6 \\
\hline $\mathrm{He}_{\mathrm{II}}^{\mathrm{a}} \ldots \ldots \ldots \ldots$ & 1831 & 1.04 & 3875 & 1.44 & 1682.7 \\
\hline $\mathrm{N}$ III] $]^{\mathrm{a}} \ldots \ldots \ldots \ldots \ldots$ & 1882 & 0.56 & 3930 & 0.80 & 1794.0 \\
\hline $\mathrm{C}$ III] $]^{\mathrm{a}} \ldots \ldots \ldots \ldots$ & 1920 & 0.86 & $\ldots$ & $\ldots$ & $\ldots$ \\
\hline $\operatorname{Mg~\Pi ^{a}} \ldots \ldots \ldots \ldots$ & 1659 & 1.73 & $\ldots$ & $\ldots$ & $\ldots$ \\
\hline
\end{tabular}

${ }^{a}$ Spectral measurements from the G230L mean spectrum. All other measurements from the G140L mean spectrum. 
FWHM $\left(\mathrm{FW}_{0.5}\right)$ in column (2) and flux contained therein $\left(F_{0.5}\right)$ in column (3). Similarly, columns (4) and (5) give the full width at $20 \%$ maximum intensity $\left(\mathrm{FW}_{0.2}\right)$ and flux therein $\left(F_{0.2}\right)$. Column $(6)$ gives the line centroid $\lambda_{0.2}^{\text {cen }}$, defined by $F_{0.2}$. No attempt has been made to deconvolve blended lines; hence, column (1) refers to the primary emission line only. In Table 1, columns (4) through (6) are empty for C III] $\lambda 1909$ and $\mathrm{Mg}$ II $\lambda 2798$ because $F_{0.2}$ is ill-defined, $\mathrm{C} \mathrm{III]} \lambda 1909$ is blended with $\mathrm{Si} \mathrm{III}] \lambda 1892$, and the estimated, extrapolated continuum near $\mathrm{Mg}$ II $\lambda 2798$ is significantly below the observed level. We note that all values in Table 1 are in the observed frame. We chose to measure line fluxes at $20 \%$ maximum intensity to minimize the effects of line blending, by measuring the primary emission-line flux only.

The uncertainties in the full width and flux measurements (Table 1) are dominated by systematic errors attributable to uncertainties in the applied absorption (where applicable) and reddening corrections. We assess the uncertainty associated with our absorption corrections by using cubic spline and linear interpolations over the Ly $\alpha \lambda 1216$ absorption feature. Figure 3 presents an illustrative example of the ambiguity in the Ly $\alpha \lambda 1216$ emission-line profile due to the different interpolation schemes. We find uncertainties of $\sim 15 \%$ and $8 \%$ for our line width and flux measurements, respectively. We do not consider the systematic uncertainty in our applied reddening corrections, given the aforementioned possibility of large internal reddening. For wavelengths $\gtrsim 1800 \AA$, systematic errors of $\sim 30 \%$ due to uncertainties in our continuum fit may be important but are not considered further.
The Ly $\alpha \lambda 1216, \mathrm{~N} v \lambda 1240$, and $\mathrm{C}$ IV $\lambda 1549$ emission lines have FWHMs of $\approx 2000 \mathrm{~km} \mathrm{~s}^{-1}$ compared to typical values of $\sim 5000 \mathrm{~km} \mathrm{~s}^{-1}$ for S1s. Their $\mathrm{FW}_{0.2}$ values are $\approx 4000$ $\mathrm{km} \mathrm{s}^{-1}$. For $\mathrm{He}$ II $\lambda 1640$, the $\mathrm{FW}_{0.5}$ and $\mathrm{FW}_{0.2}$ estimates are $\approx 1000$ and $3000 \mathrm{~km} \mathrm{~s}^{-1}$, respectively (from the mean G140L spectrum).

The emission-line profiles appear symmetric about their systemic wavelengths, but this is difficult to quantify because of contaminating emission, such as $[\mathrm{O}$ III $] \lambda 1664$ in the red wing of He II $\lambda 1640$, and absorption features affecting many of the line profiles. The centroids, $\lambda_{0.2}^{\text {cen }}$, of the observed emission-line fluxes of Ly $\alpha \lambda 1216, \mathrm{~N} v \lambda 1240$, $\mathrm{Si}$ IV $+\mathrm{O}$ IV] $\lambda 1400, \mathrm{C}$ IV $\lambda 1549, \mathrm{~N}$ III] $\lambda 1750$, and $\mathrm{Mg}$ II $\lambda 2798$ are consistent with those expected based on the systemic redshift $z=0.0247$, given the 1.2 and $3.2 \AA$ spectral resolutions of the G140L and G230L gratings. The [O I] $\lambda 1336$ and $\mathrm{N}$ IV] $\lambda 1523$ emission-line flux centroids are redshifted and blueshifted by 2.5 and $1.6 \AA$, respectively. The [O I] $\lambda 1336 \lambda_{0.2}^{\text {cen }}$ is possibly biased by $\mathrm{S}$ II $\lambda 1340$ emission. The He II $\lambda 1640$ emission-line flux centroid is blueshifted by $1.3 \AA\left(\approx 230 \mathrm{~km} \mathrm{~s}^{-1}\right)$. The He II $\lambda 1640 \lambda_{0.2}^{\text {cen }}$ measured from the G230L mean spectrum is most likely biased by [O III] $\lambda 1664$ contamination. Figure 4 presents the mean Ly $\alpha$ $\lambda 1216, \mathrm{C}$ IV $\lambda 1549$, and $\mathrm{Mg}$ II $\lambda 2798$ emission-line profiles as a function of velocity, with the above-defined continuum fit subtracted. The emission-line profile amplitudes have been normalized to unity. The high-ionization lines of $\operatorname{Ly} \alpha \lambda 1216$ and C IV $\lambda 1549$ and the low-ionization line of $\mathrm{Mg}$ II $\lambda 2798$ do not appear notably blueshifted or redshifted relative to systemic velocities. These results are discussed in $\S 6.1$.

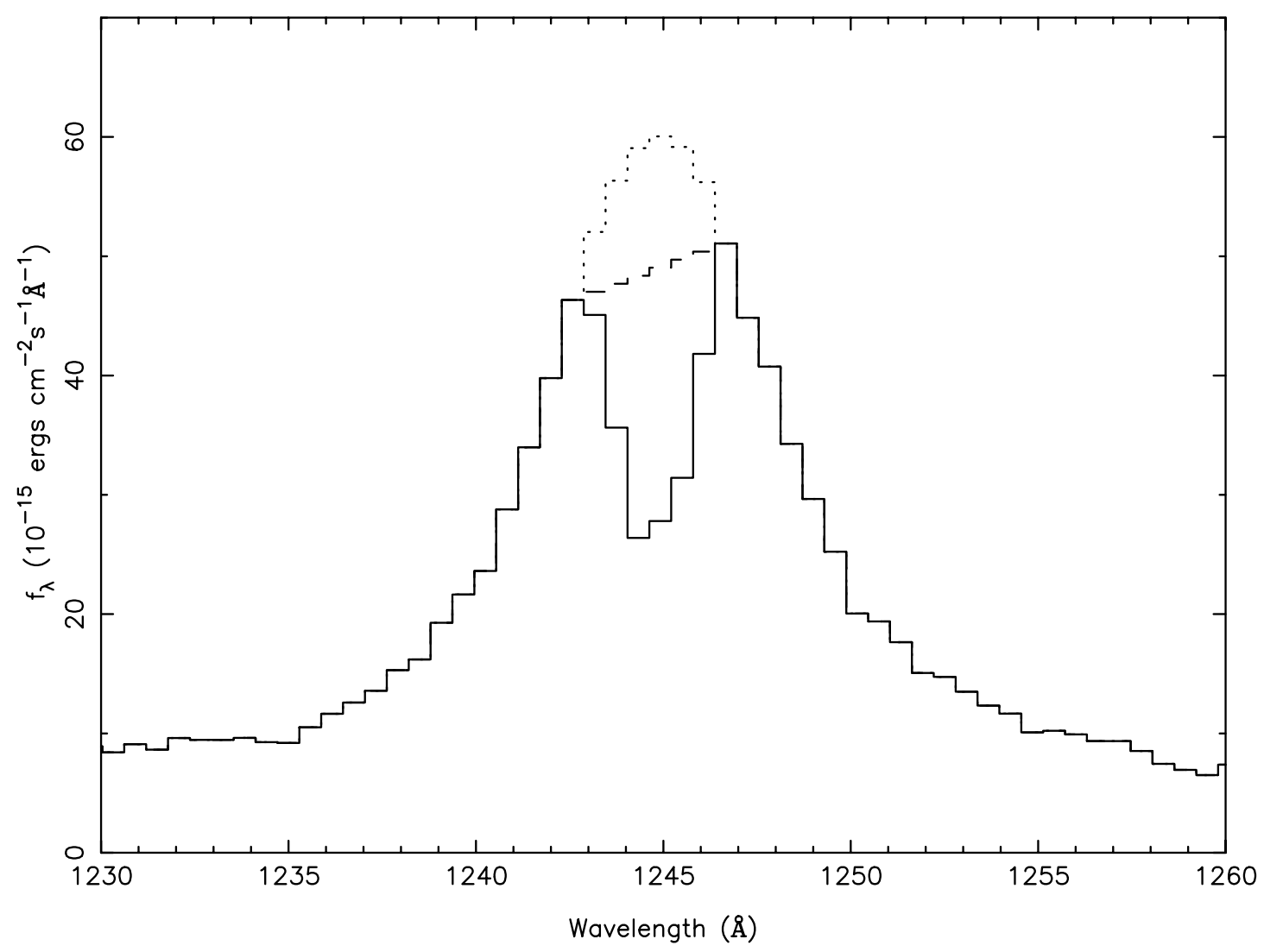

FIG. 3.-Illustrative example of the ambiguity in the Ly $\alpha \lambda 1216$ emission-line profile and, hence, spectral line measurements (for which absorption corrections have been made) of $\S 3$, owing to different interpolation schemes. The solid line histogram presents the Ly $\alpha \lambda 1216$ emission-line profile, and the dashed and dotted histograms detail the linear and cubic spline interpolation corrections over the absorption feature. 


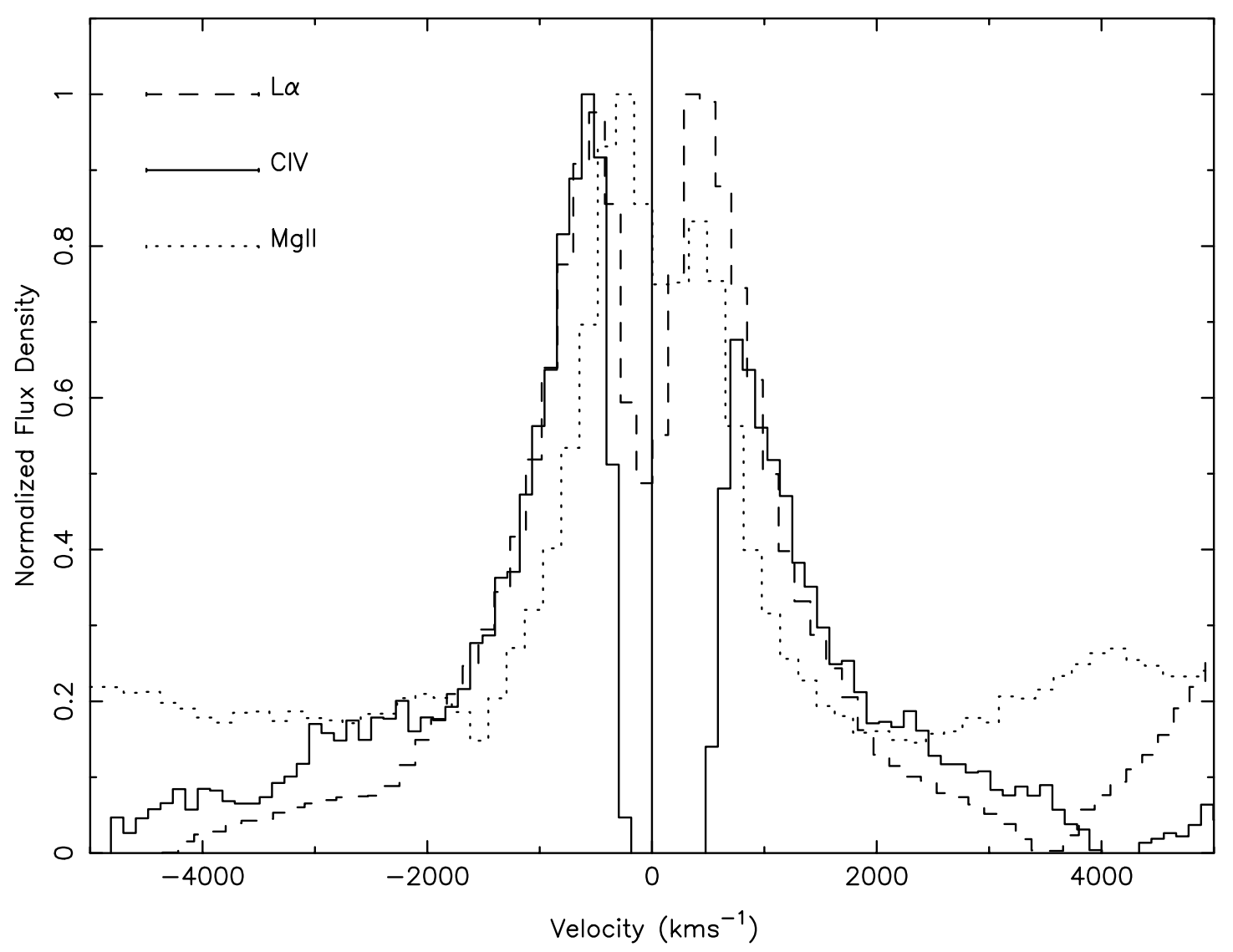

FIG. 4.-Mean Ly $\alpha \lambda 1216$, C IV $\lambda 1549$, and Mg II $\lambda 2798$ emission-line profiles of Figs. 1 and 2 as a function of velocity. The emission-line profile amplitudes have been normalized to unity. The high-ionization lines of $L y \alpha \lambda 1216$ and $C$ IV $\lambda 1549$ and the low-ionization line of Mg II $\lambda 2798$ do not appear notably blueshifted or redshifted relative to systemic velocities.

We find some significant differences between emissionline flux ratios in Ark 564 relative to those of more typical S1s. From Table 1, measured line ratios for Ark 564 are $(\mathrm{C}$ IV/Ly $\alpha)=0.27(1.09),(\mathrm{C} \mathrm{III]} / \mathrm{Ly} \alpha)^{*}=0.16(0.13),(\mathrm{C} \mathrm{III]} /$ $\left.\left.\begin{array}{lllll}\mathrm{C} & \mathrm{IV}\end{array}\right)^{*}=0.65 \quad(0.12), \quad(\mathrm{Si} \quad \mathrm{IV}+\mathrm{O} \quad \mathrm{IV}] / \mathrm{C} \quad \mathrm{IV}\right)=0.48$ $(0.07),(\mathrm{Si}$ IV + O IV $] / \mathrm{Ly} \alpha)=0.13(0.08),(\mathrm{Mg} \mathrm{II} / \mathrm{Ly} \alpha)^{*}=$ $0.33(0.22)$, and $(\mathrm{He}$ II $/ \mathrm{C} \mathrm{IV})=0.78(0.09)$. The line ratios in parentheses are those for the S1 NGC 5548, taken from Clavel et al. (1991). Line ratios with an asterisk denote those (for Ark 564) estimated using the fluxes at 50\% maximum intensity, compared to $20 \%$ for the others. This was necessary because, as mentioned previously, $F_{0.2}$ is ill-defined for $\mathrm{C}$ III] $\lambda 1909$ and $\mathrm{Mg}$ II $\lambda 2798$. The uncertainties in these line ratios are dominated by systematic effects, discussed above, and are on the order of $25 \%$. This error estimate includes an $\sim 15 \%$ bias owing to excluding flux in the line wings. For line ratios that include C III] $\lambda 1909$ and $\mathrm{Mg}$ II $\lambda 2798$, the error estimate may be notably underestimated on account of the continuum fit uncertainties described above. Our emission-line ratios show Ark 564 exhibits weaker C IV $\lambda 1549$ and stronger $\mathrm{Si}$ IV $+\mathrm{O}$ IV] $\lambda 1400$ emission compared to NGC 5548. These results are consistent with earlier results of Wilkes et al. (1999) and Kuraszkiewicz et al. (2000). We note our $\mathrm{Mg} \mathrm{II} / \mathrm{Ly} \alpha$ ratio of 0.33 is notably larger than the mean value of 0.05 derived from the small sample of NLS1s by Kuraszkiewicz et al. (2000). This is probably largely attributable to different continuum level estimates near to $\mathrm{Mg}$ II $\lambda 2798$.

Finally, we checked archival IUE observations of Ark 564 to assess whether it was in a comparatively low or high flux state. Figure 5 presents the IUE SWP observation of 1984 Jan 17. For comparison purposes, our mean G140L spectrum of Figure 1 is overlaid as the thicker line. Both spectra have been corrected for Galactic reddening as detailed above. The continuum and emission-line fluxes are in qualitative agreement. In particular, the Ly $\alpha \lambda 1216$ and $\mathrm{N} v \lambda 1240$ line profiles are very similar and suggest negligible emission-line flux differences between the 1984 and 2000 observations. Other IUE observations from 1981 are also in qualitative agreement with our observations.

\section{CONTINUUM VARIABILITY CHARACTERISTICS}

\subsection{Light Curves}

We select five nominal continuum bands by visual inspection of the mean and rms spectra presented in Figures 1 and 2: $1350-1380 \AA$, $1460-1500 \AA, 1620-1660 \AA, 2070-2130 \AA$, and 2960-3040 $\AA$, henceforth referred to by their mean observed wavelengths $1365,1480,1640,2100$, and $3000 \AA$, respectively. We reiterate that each of these continuum bands overestimates the true continuum level on account of various contaminants $(\S 3)$. These bands are not notably biased by emission-line variability and therefore provide good approximations for the continuum variability at these wavelengths. Light curves, describing the continuum variability during the 60 day monitoring period, are presented in Figure 6, with the continuum bands as labeled. We note our observed continuum flux measurements are not corrected for reddening.

All the continuum regions show the same qualitative 


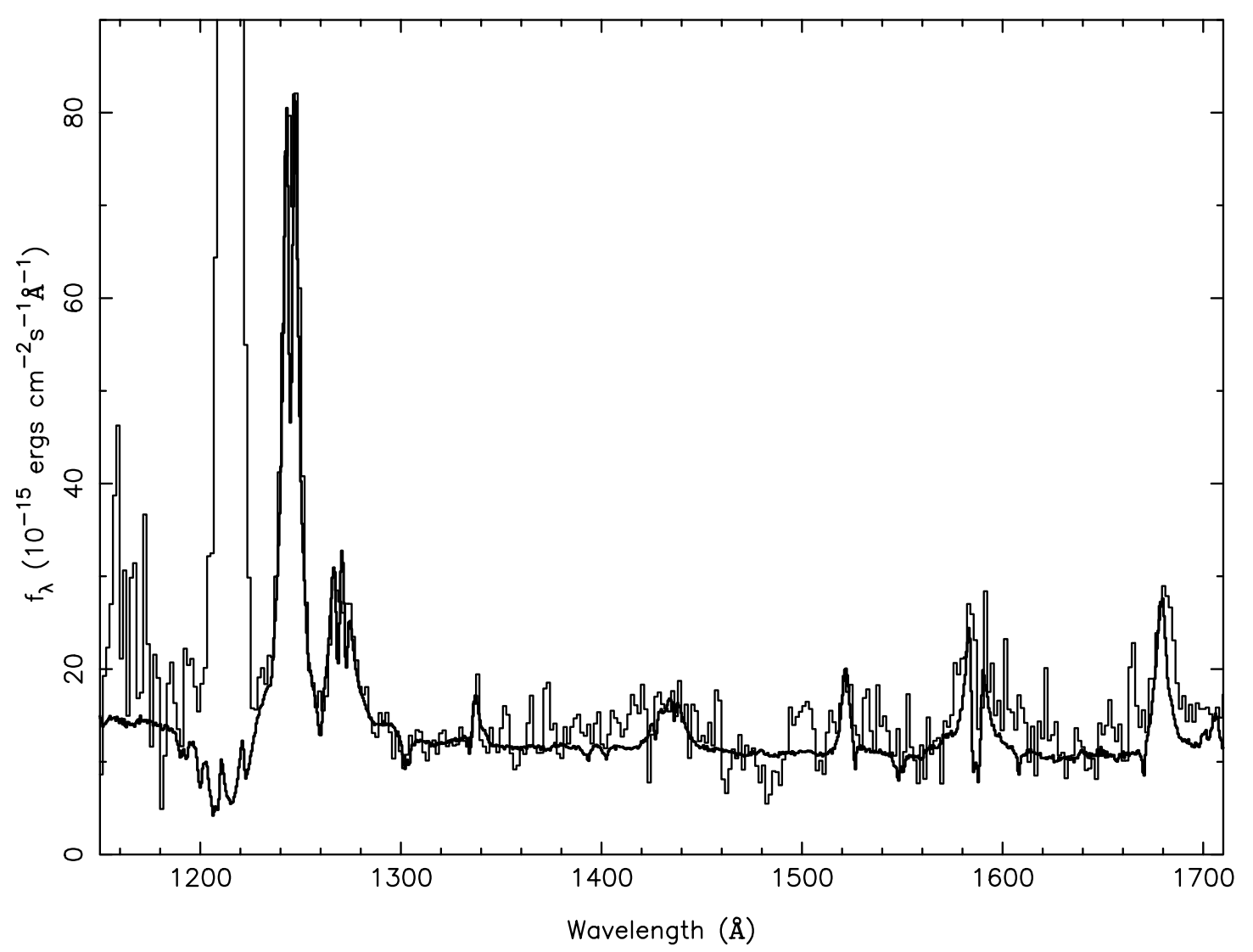

Fig. 5.-1984 Jan 17 IUE SWP observation of Ark 564. For purposes of comparison, our mean G140L spectrum of Fig. 1 is overlaid as the thicker line. These spectra have been corrected for Galactic reddening using $E(B-V)=0.06 \mathrm{mag}$. The continuum and emission-line fluxes are in qualitative agreement.

behavior. The $1365 \AA$ variations may be described by an increase in flux of $\sim 20 \%$ during the first 15 days, followed by a decrease of $\sim 16 \%$ over the next 5 days. These variations are followed by two flares at about JD $-2,450,000 \approx 1697$ and 1711 . The rising trough-to-peak variations are about $18 \%$ in $\sim 3$ days, with similar declining peak-to-trough variations occurring over longer timescales of several days. Both flare events appear to be asymmetric, the later one more so and of longer duration. Between these two events was a relatively quiescent period of about 5 days. Thereafter, the variations are less pronounced, at about the $5 \%$ level. For the longer wavelength variations, at 2100 and $3000 \AA$, the rms variations are of reduced amplitude with broader asymmetric flare profiles. The peaks of the $3000 \AA$ flares are delayed, relative to those at $1365 \AA$, by $1-2$ days. The contemporaneous $A S C A$ observations exhibit larger amplitude-correlated variations (including similar but narrower twin flare events), possibly delayed by about 0.4 days (Papers I and III). We note the continuum variations may be slightly undersampled during the intensive monitoring period of $\mathrm{JD}-2,450,000 \approx 1694-1734$ and are definitely undersampled for prior times, given that $\sim 10 \%$ flux amplitude changes on timescales of 1 day are evident (see below).

We characterize the $1365-3000 \AA$ variations by measuring two common variability parameters: the ratio of the maximum to minimum flux $R_{\max }$ and the amplitude of the intrinsic variability relative to the mean flux $F_{\text {var }}$. The latter is corrected for the measurement errors $\epsilon$,

$$
F_{\text {var }}=\frac{1}{\bar{F}} \sqrt{\left(\sigma_{\mathrm{F}}^{2}-\Delta^{2}\right)},
$$

where $\bar{F}, \sigma_{\mathrm{F}}$, and $\Delta^{2}=(1 / N) \sum_{i=1}^{N} \epsilon_{i}^{2}$ are, respectively, the standard mean flux, rms flux, and mean square of the measurement errors, with $N=46$ the number of data points in the light curve (Edelson, Krolik, \& Pike 1990; RodríguezPascual et al. 1997b). The results are presented in Table 2 for each of the light curves, as listed in column (1). Columns (2)-(5) give, respectively, the mean flux $\bar{F}$, the rms flux $\sigma_{\mathrm{F}}$, $F_{\text {var }}$, and $R_{\max }$. Both $F_{\text {var }}$ and $R_{\max }$ are potentially biased by constant flux components (e.g., Fe II emission). However, these effects are likely to be small. On timescales of about 60 days, the fractional amplitude of the intrinsic variations is

TABLE 2

CONTINUUM AND EMISSION-LINE VARIABILITY CHARACTERISTICS

\begin{tabular}{ccccc}
\hline \hline $\begin{array}{c}\text { Light Curve } \\
(1)\end{array}$ & $\begin{array}{c}\bar{F}^{\mathrm{a}} \\
(2)\end{array}$ & $\begin{array}{c}\sigma_{F}{ }^{\mathrm{a}} \\
(3)\end{array}$ & $\begin{array}{c}F_{\text {var }} \\
(4)\end{array}$ & $\begin{array}{c}R_{\max } \\
(5)\end{array}$ \\
\hline $1365 \AA \ldots \ldots \ldots$. & 7.21 & 0.44 & 0.061 & 1.31 \\
$1480 \AA \ldots \ldots \ldots \ldots$ & 6.99 & 0.46 & 0.065 & 1.33 \\
$1640 \AA \ldots \ldots \ldots$. & 6.98 & 0.46 & 0.064 & 1.33 \\
$2100 \AA \ldots \ldots \ldots$. & 5.82 & 0.30 & 0.051 & 1.28 \\
$3000 \AA \ldots \ldots \ldots$. & 6.48 & 0.25 & 0.037 & 1.19 \\
$\operatorname{Ly} \alpha \lambda 1216^{\mathrm{b}} \ldots \ldots$. & 16.95 & 0.30 & 0.015 & 1.07 \\
$\mathrm{Ly} \alpha \lambda 1216^{\mathrm{c}} \ldots \ldots$. & 29.10 & 0.42 & 0.012 & 1.06 \\
\hline
\end{tabular}

${ }^{\text {a }}$ Units are $10^{-15} \mathrm{ergs} \mathrm{cm}^{-2} \mathrm{~s}^{-1} \AA^{-1}$ for continuum fluxes and $10^{-14} \mathrm{ergs} \mathrm{cm}{ }^{-2} \mathrm{~s}^{-1}$ for the line fluxes. All light curves have $N=46$ data points.

${ }^{b}$ Ly $\alpha \lambda 1216$ variations between $1240-1243 \AA$ and 1247-1250 A.. (See $\S 5$ for further details.)

${ }^{c}$ Ly $\alpha \lambda 1216$ variations between 1240 and $1250 \AA ̊$. (See $\S 5$ for further details.) 


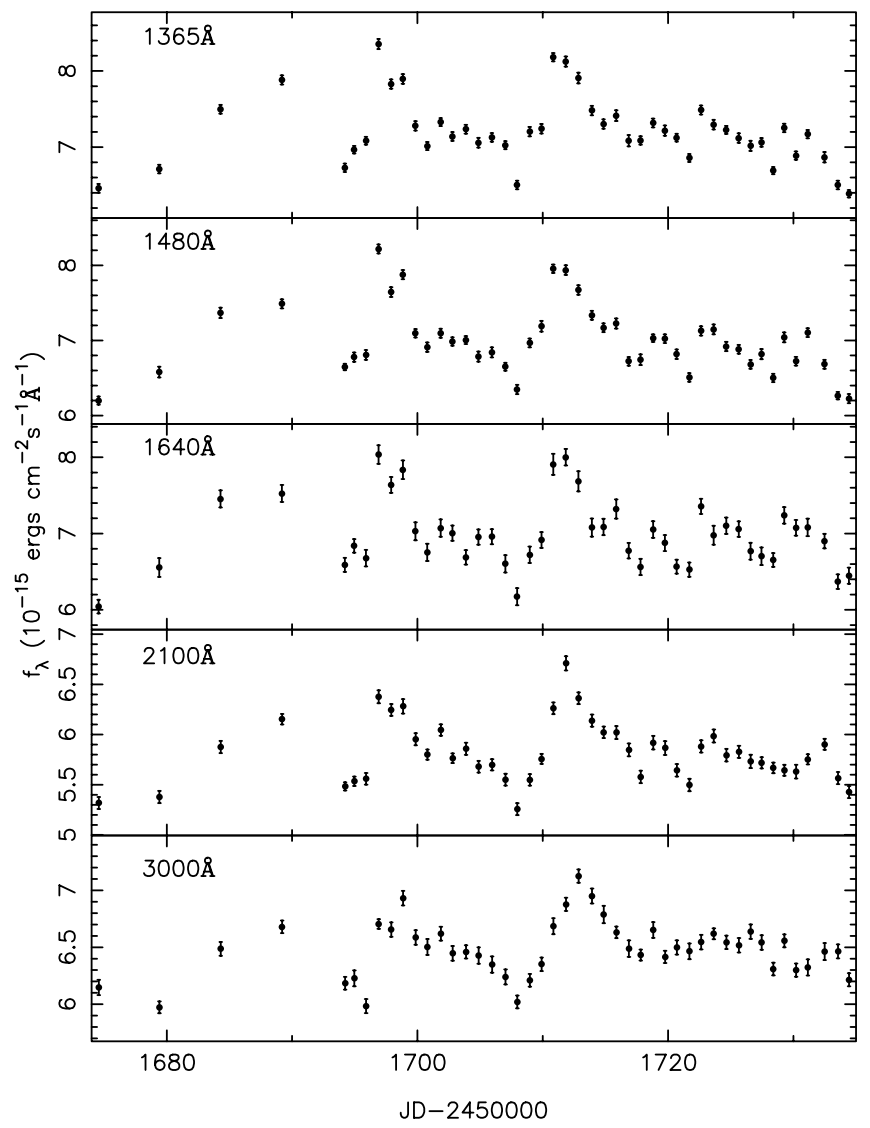

FIG. 6.-Observed continuum light curves for the 60 day monitoring period, with mean observed wavelength as labeled. The amplitude of the intrinsic rms variations is about $6 \%$, and they all exhibit the same qualitative behavior.

essentially constant between 1365 and $2100 \AA$ at about $6 \%$, with the full range of variations $R_{\max }-1 \approx 0.31$. At $3000 \AA$ the fractional variability amplitude is about $4 \%$, with the full range of variations $R_{\max }-1 \approx 0.19$. This level of intrinsic UV variability is about a factor of 3 less than that found in typical S1s, which typically display fractional flux variations $F_{\text {var }} \approx 18 \%$ on timescales of 60 days (Collier, Peterson, \& Horne 2001).

The 1365-1640 $\AA$ continuum variations show evidence for $\sim 10 \%$ flux amplitude changes on timescales of about 1 day, as seen in the events beginning at about JD $-2,450,000 \approx 1696,1710$, and 1707 , and suggest a fraction of the UV continuum-emitting region must come from a compact region of $\lesssim 1$ 1t-day in size. The $2100-3000 \AA$ variations show evidence for reduced flux amplitude changes of $\sim 5 \%$ on similar timescales. For comparison, in S1s far-UV variations of about $5 \%$ rms occur on similar timescales (e.g., Korista et al. 1995; Welsh et al. 1998). These faster, larger amplitude variations suggest NLS1s (at least Ark 564) exhibit more variability power on short (day) timescales. We confirm this through an autocorrelation analysis. Figure 7 presents the Ark $5641365 \AA$ (solid line) and NGC 7469 (an S1) $1315 \AA$ (dotted line) autocorrelation functions (ACFs). The relative steepness of the $1365 \AA$ ACF (compared to the $1315 \AA$ ACF) indicates Ark 564's fluctuation power density spectrum is flatter than that for NGC 7469 and therefore exhibits more power on short timescales. We note Pounds et al. (2001) have shown that the X-ray variations in Ark 564 are faster than those of typical S1s.
The FWHM of the ACFs are 3.27 and 4.93 days for Ark 564 and NGC 7469, respectively, and are indicative of characteristic UV variability timescales. By assuming the mass ratio of the sources is determined by the variability timescale ratio of 0.66 , we estimate the mass of Ark 564 to be $M \sim 5 \times 10^{6} M_{\odot}$, given a mass estimate for NGC 7469 of $8 \times 10^{6} M_{\odot}$ (Wandel et al. 1999). This mass estimate (for Ark 564) is in good agreement with that derived from our emission-line reverberation results, discussed in $\S 6.2$.

\subsection{Cross-Correlation Analysis}

The continuum light curves of Figure 6 clearly display correlated variations between 1365 and $3000 \AA$. The peaks and troughs of the flare events at JD $-2,450,000 \approx 1697$ and 1711 occur approximately simultaneously; as mentioned above, the $3000 \AA$ flare peaks appear delayed by about 1-2 days relative to those at shorter wavelengths. Differences between the light curves are also evident; for example, decay timescales for the two flares appear comparatively longer at wavelengths $\geq 2100 \AA$, and short (day) timescale $1365 \AA$ variations appear washed out at $3000 \AA$. In order to quantify the nature of the correlations between continuum variations, we undertook a cross-correlation analysis. Two algorithms were employed to compute crosscorrelation functions (CCFs), the interpolated CCF (ICCF) of Gaskell \& Sparke (1986) as implemented by White \& Peterson (1994), and the $Z$-transformed discrete correlation function (ZDCF) algorithm of Alexander (1997). For our data, the results recovered by the two algorithms are in agreement.

Figure 8 shows the CCFs obtained by cross-correlating each of the four longer wavelength continuum light curves with the $1365 \AA$ light curve. The solid line and data points with error bars show the ICCF and ZDCF CCFs, respectively, and are in good agreement. The $1365-3000 \AA$ variations are highly correlated as evidenced by maximum values of the cross-correlation coefficients $r_{\max } \approx 0.9$. The 1480 $2100 \AA$ CCFs all peak at about zero lag, thereby suggesting these continuum variations occur quasi-simultaneously. The $3000 \AA$ CCF peaks away from zero at about 0.5 days. The 2100 and $3000 \AA$ CCFs appear asymmetric, compared to the 1480 and $1640 \AA$ CCFs, and suggest their responses extend over a larger range of positive delays. Figure 9 presents similar CCFs for four optical continuum regions:

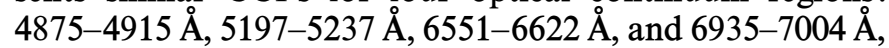
henceforth referred to by the observed wavelengths 4900 , 5200,6600 , and $6900 \AA$, respectively (for consistency with Paper III). The optical data are from Paper III. These CCFs have been computed as described above. The optical variations are correlated with those at UV wavelengths with $r_{\max } \approx 0.5$. The decreasing maximum correlation coefficient with optical wavelength is likely due to progressively less coherent, time delay smeared responses and/or increasing noise dilution (see Paper III, Table 4). The optical CCFs clearly peak away from zero at about 2 days.

Table 3 summarizes our cross-correlation results. Column (1) lists the light curve that has been crosscorrelated with the $1365 \AA$ continuum light curve. Columns (2) and (3) give the centroid of the CCF $\tau_{\text {cen }}$, as determined by the ICCF and ZDCF algorithms. The ICCF centroid is calculated over all points above 0.8 times the maximum cross-correlation coefficient $r_{\max }$, whereas the ZDCF centroid is computed from all points near the peak with a cross-correlation amplitude above half that of the peak. 


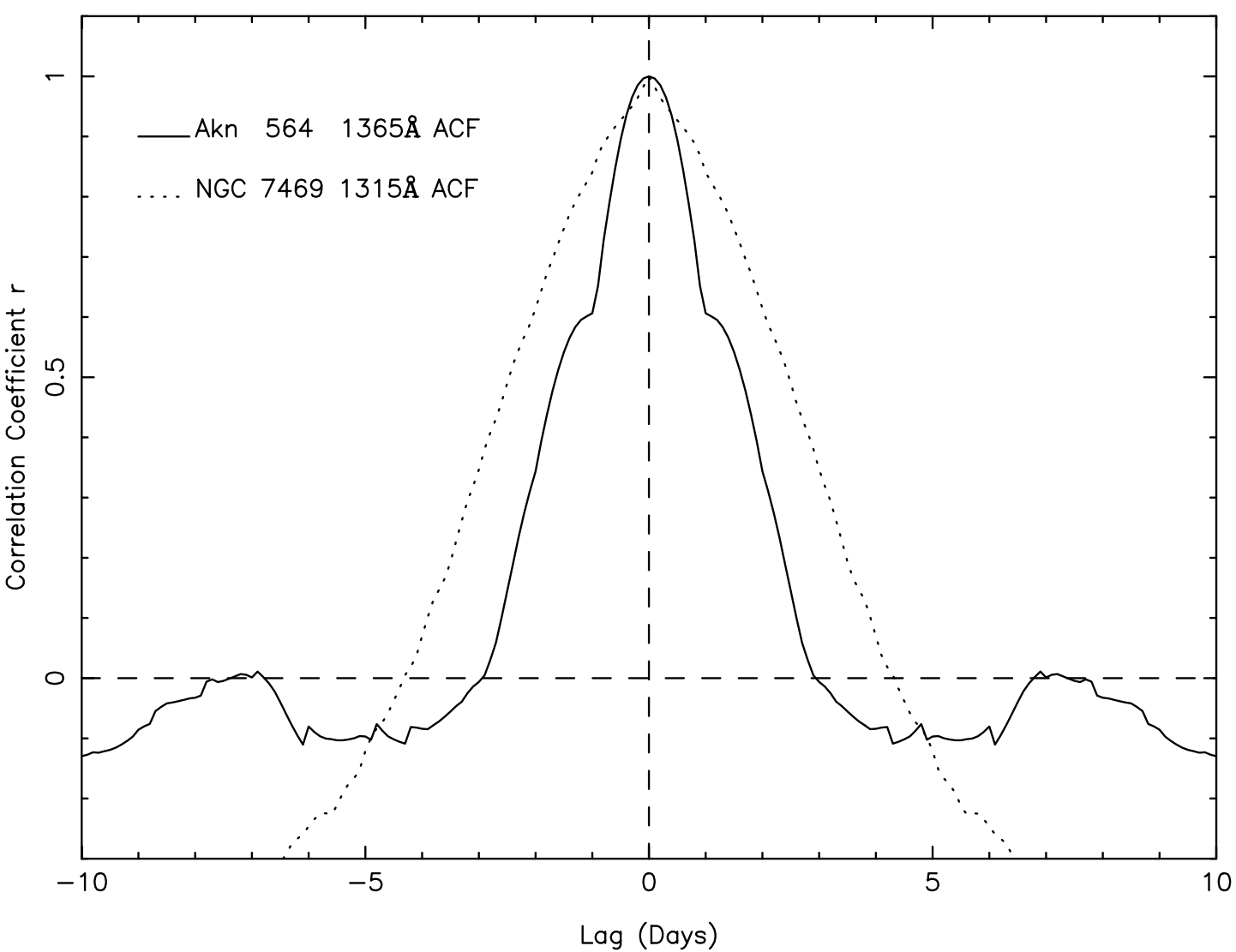

FIG. 7.-Ark $5641365 \AA$ (solid line) and NGC 7469 (an S1) $1315 \AA$ (dotted line) autocorrelation functions (ACFs), respectively. The relative steepness of the $1365 \AA$ ACF (compared to the $1315 \AA$ ACF) indicates Ark 564's fluctuation power density spectrum is flatter than that for NGC 7469 and therefore exhibits more power on short timescales. The FWHM of the ACFs are 3.27 and 4.93 days for Ark 564 and NGC 7469, respectively, and are indicative of characteristic UV variability timescales.

Columns (4) and (5) report the time delay $\tau_{\text {peak }}$ measured from the peak of the CCF. Columns (6) and (7) give $r_{\max }$ for the ICCF and ZDCF, respectively. Column (8) details the FWHM of the ICCF. The reported errors on $\tau_{\text {peak }}$ and $\tau_{\text {cen }}$ for the ICCF are the $1 \sigma$ uncertainties as determined by the model-independent quasi-bootstrap and flux randomiza- tion method of Peterson et al. (1998). We find no evidence for lags between the 1365 and $1640 \AA$ light curves, as evidenced by peak and centroid lag measurements consistent with zero. The 2100 and $3000 \AA$ variations, however, lag those at $1365 \AA$ by $\sim 0.3$ and 1.0 day, respectively. Here and hereafter, we note the centroid lags since they are a less

TABLE 3

Cross-CORRELATION ReSUlts

\begin{tabular}{|c|c|c|c|c|c|c|c|}
\hline \multirow[b]{2}{*}{$\begin{array}{l}\text { BAND } \\
\text { (1) }\end{array}$} & \multicolumn{2}{|c|}{$\begin{array}{c}\tau_{\text {cen }} \\
\text { (days) }\end{array}$} & \multicolumn{2}{|c|}{$\begin{array}{c}\tau_{\text {peak }} \\
\text { (days) }\end{array}$} & \multicolumn{2}{|c|}{$r_{\max }$} & \multirow{2}{*}{$\begin{array}{c}\begin{array}{c}\text { FWHM } \\
\text { (days) }\end{array} \\
\begin{array}{c}\text { ICCF } \\
(8)\end{array}\end{array}$} \\
\hline & $\begin{array}{c}\text { ICCF } \\
(2)\end{array}$ & $\begin{array}{c}\text { ZDCF } \\
\text { (3) }\end{array}$ & $\begin{array}{c}\text { ICCF } \\
\text { (4) }\end{array}$ & $\begin{array}{c}\text { ZDCF } \\
(5)\end{array}$ & $\begin{array}{c}\text { ICCF } \\
(6)\end{array}$ & $\begin{array}{c}\text { ZDCF } \\
\text { (7) }\end{array}$ & \\
\hline $1480 \AA ̊ . . . \ldots \ldots . .$. & $-0.0_{-0.2}^{+0.2}$ & 0.0 & $0.0 \pm 0.0$ & $0.0 \pm 0.4$ & 1.0 & 1.0 & 3.3 \\
\hline $1640 \AA ̊ \ldots \ldots \ldots$ & $0.1_{-0.2}^{+0.2}$ & 0.2 & $0.0 \pm 0.1$ & $0.0 \pm 0.4$ & 0.9 & 1.0 & 3.0 \\
\hline $2100 \AA ̊ . . . \ldots \ldots . . .$. & $0.3_{-0.2}^{+0.2}$ & 0.3 & $0.3_{-0.1}^{+0.2}$ & $0.0_{-0.4}^{+0.4}$ & 0.9 & 0.9 & 3.0 \\
\hline $3000 \AA ̊ . . . . . . .$. & $1.0_{-0.3}^{+0.4}$ & 0.7 & $0.5_{-0.0}^{+1.1}$ & $0.0_{-0.4}^{+1.4}$ & 0.8 & 0.7 & 3.5 \\
\hline $4900 \AA ̊ . . . \ldots \ldots$ & $1.8_{-0.4}^{+0.5}$ & 1.8 & $2.2_{-1.0}^{+0.2}$ & $2.7 \pm 1.3$ & 0.7 & 0.6 & 3.6 \\
\hline $5200 \AA ̊ . . . \ldots \ldots$ & $1.8_{-0.4}^{+0.6}$ & 1.7 & $1.4_{-0.1}^{+1.0}$ & $1.7_{-1.0}^{+2.5}$ & 0.6 & 0.5 & 3.7 \\
\hline $6600 \AA ̊ . . . \ldots \ldots$ & $2.2_{-8.3}^{+1.7}$ & 2.3 & $2.5_{-8.8}^{+1.8}$ & $2.1_{-1.5}^{+0.8}$ & 0.4 & 0.5 & 3.8 \\
\hline $6900 \AA ̊ . . . \ldots \ldots$ & $2.6_{-5.1}^{+1.6}$ & 2.4 & $3.0_{-6.4}^{+1.8}$ & $2.7_{-1.8}^{+1.3}$ & 0.4 & 0.3 & 3.7 \\
\hline $\operatorname{Ly} \alpha \lambda 1216^{\mathrm{a}} \ldots \ldots$ & $0.9_{-1.2}^{+0.4}$ & 0.4 & $0.7_{-1.1}^{+0.6}$ & $1.0_{-1.7}^{+0.6}$ & 0.6 & 0.5 & 5.0 \\
\hline $\operatorname{Ly} \alpha \lambda 1216^{b} \ldots \ldots$ & $2.8_{-2.3}^{+0.0}$ & 1.9 & $2.7_{-2.2}^{+0.0}$ & $1.0_{-1.4}^{+1.8}$ & 0.5 & 0.4 & 3.6 \\
\hline
\end{tabular}

${ }^{\mathrm{a}}$ Ly $\alpha \lambda 1216$ variations between $1240-1243 \AA$ And $1247-1250 \AA$ A See $\S 5$ for further details.

${ }^{b}$ Ly $\alpha \lambda 1216$ variations between $1240-1250 \AA$ A. See $\S 5$ for further details. 


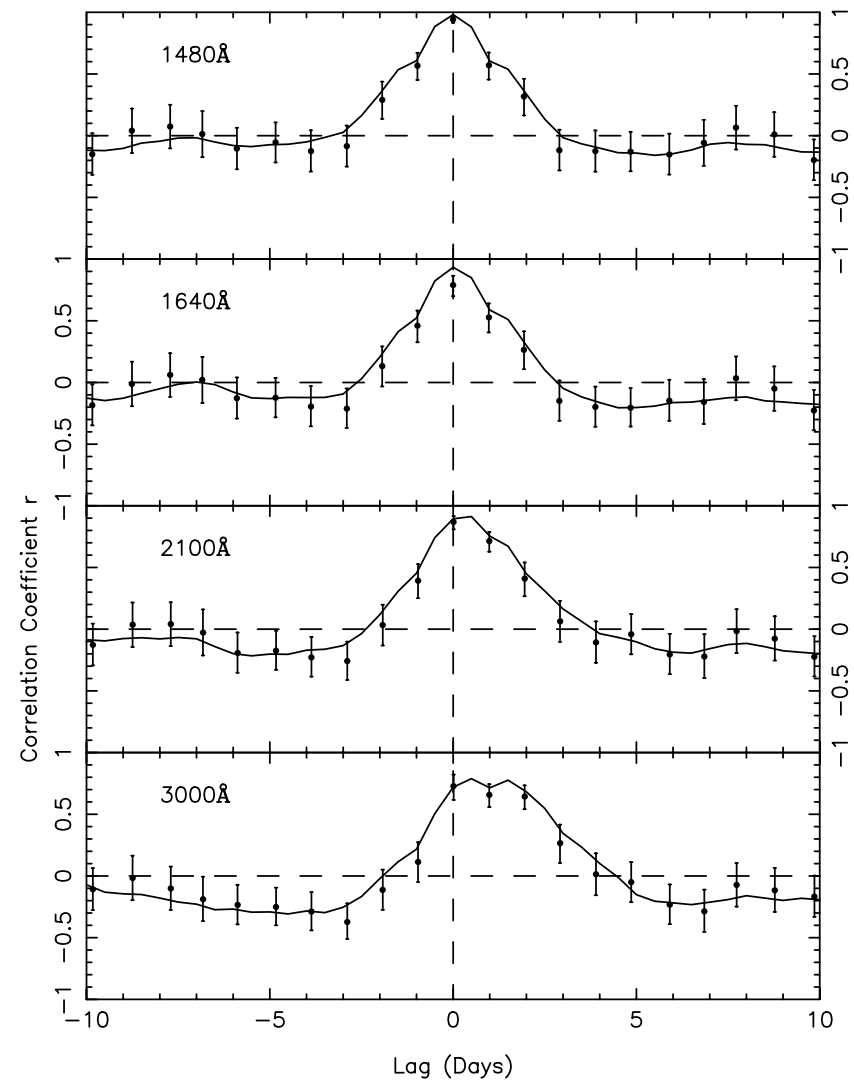

FIG. 8.-CCFs for four UV continuum regions, mean observed wavelengths as labeled. The solid line and data points with error bars detail the ICCF and ZDCF CCFs, respectively, and are in good agreement. The $1480-3000 \AA$ variations are strongly correlated with those at $1365 \AA$ as evidenced by maximum cross-correlation coefficients of $r_{\max } \approx 0.9$. The 1480-2100 A variations occur quasi-simultaneously, since their CCFs all peak at about zero lag. The $3000 \AA$ CCF peaks at about 0.5 days.

biased estimator of the size of the responding emission region. These lags are greater than 0 at $94 \%$ and $99 \%$, respectively. The optical variations at $\sim 5000 \AA$ lag behind those at $1365 \AA$ by about 2 days at no less than $99 \%$ confidence. The optical variations at wavelengths longer than $6000 \AA$ appear to lag those at $1365 \AA$ by a similar amount, although statistically the suggested lags are not significantly different from zero on account of their very low amplitude $(\sim 1 \%)$ variability, which is comparable to the measurement errors.

We investigate these wavelength-dependent continuum lags further by binning the $46 \mathrm{G} 140 \mathrm{~L}$ and G230L spectra (covering 1150-3140 A) into $40 \AA$ bins and forming light curves on the basis of the total flux in each bin. We crosscorrelate (using the ICCF) each of these light curves with the $1365 \AA$ light curve. The results are presented in Figure 10. In the top panel, the histogram plot shows the centroid lag for each bin as a function of the bin wavelength, thus producing a "lag spectrum." The corresponding values of $r_{\max }$ are shown in the lower panel. Again, the error bars for each bin in the upper panel were determined by the method of Peterson et al. (1998). The solid line represents the best-fit function $\tau \propto\left(\lambda^{\gamma}-\lambda_{0}^{\gamma}\right)$, with $\lambda_{0}=1365 \AA, \gamma=2.4 \pm 0.1$, and reduced chi-squared $\chi_{v}^{2}=0.07$ for $v=50$ degrees of freedom (dof) to the lag measurements. The small value of $\chi_{v}^{2} \ll 1$ suggests the lag uncertainties may be overestimated. There is a clear trend of increasing lag with wavelength

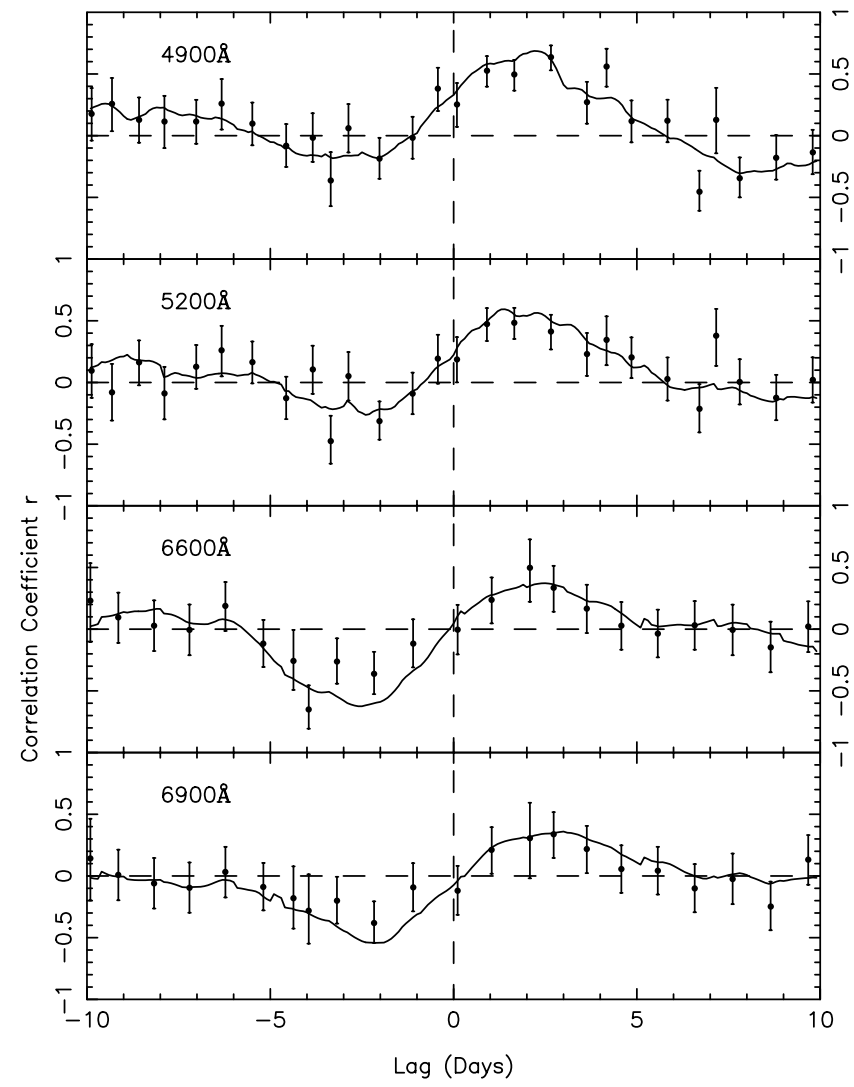

FIG. 9.-CCFs for four optical continuum regions, with nominal observed wavelengths as labeled. All CCFs have been computed with respect to the variations at $1365 \AA$. The solid line and data points with error bars detail the ICCF and ZDCF CCFs, respectively, and are in good agreement. The 4900-6900 $\AA$ variations are correlated with those at 1365 $\AA$, as evidenced by maximum correlation coefficients of $r_{\max } \approx 0.5$. Moreover, they are delayed by about 2 days with respect to those at UV wavelengths.

throughout the UV; the variations at $3100 \AA$ lag those at $1365 \AA$ by about 0.9 days. The lag measurements for $\lambda \gtrsim$ $2050 \AA$ are nonzero at no less than $90 \%$ confidence. For wavelengths shorter than $2050 \AA$, the lag measurements are consistent with zero delay. The $1150-3140 \AA$ variations are well correlated with $r_{\max } \approx 0.9$. In the region where the G140L and G230L spectra overlap (i.e., 1600-1700 $\AA$ ) the lag measurements are in good agreement. The lag spectrum is reasonably smooth with no clear positive deviations near the emission-line wavelengths, as would be the case if the emission-line response timescales were measurably larger. Similarly, there are no negative dips in the maximum correlation coefficient spectrum, with the possible exception near $\mathrm{Mg}$ II $\lambda 2798$. These results support the negligible presence of emission-line variability to be discussed in $\S 5$. We note the dip in the maximum correlation coefficient at about $1700 \AA$ is due to calibration uncertainties in the G230L spectra. Similarly, the slight depression in $r$ at $\lesssim 1200 \AA$ is due to calibration errors in the G140L spectra. We note the two bins with the largest centroid lag errors are those contaminated by $\mathrm{Ly} \alpha \lambda 1216$ and $\mathrm{Mg}$ II $\lambda 2798$.

We use the optical lag measurements (Table 3) to extend the UV lag spectrum. The UV/optical lag spectrum is presented in Figure 11. The solid line represents the best-fit function $\tau \propto\left(\lambda^{\gamma}-\lambda_{0}^{\gamma}\right)$, with $\lambda_{0}=1365, \gamma=1.3 \pm 0.1$, and $\chi_{v}^{2}=0.09$ for $v=54$ dof. The dotted line represents the bestfit function to the UV data alone (as in Fig. 10), i.e., with 


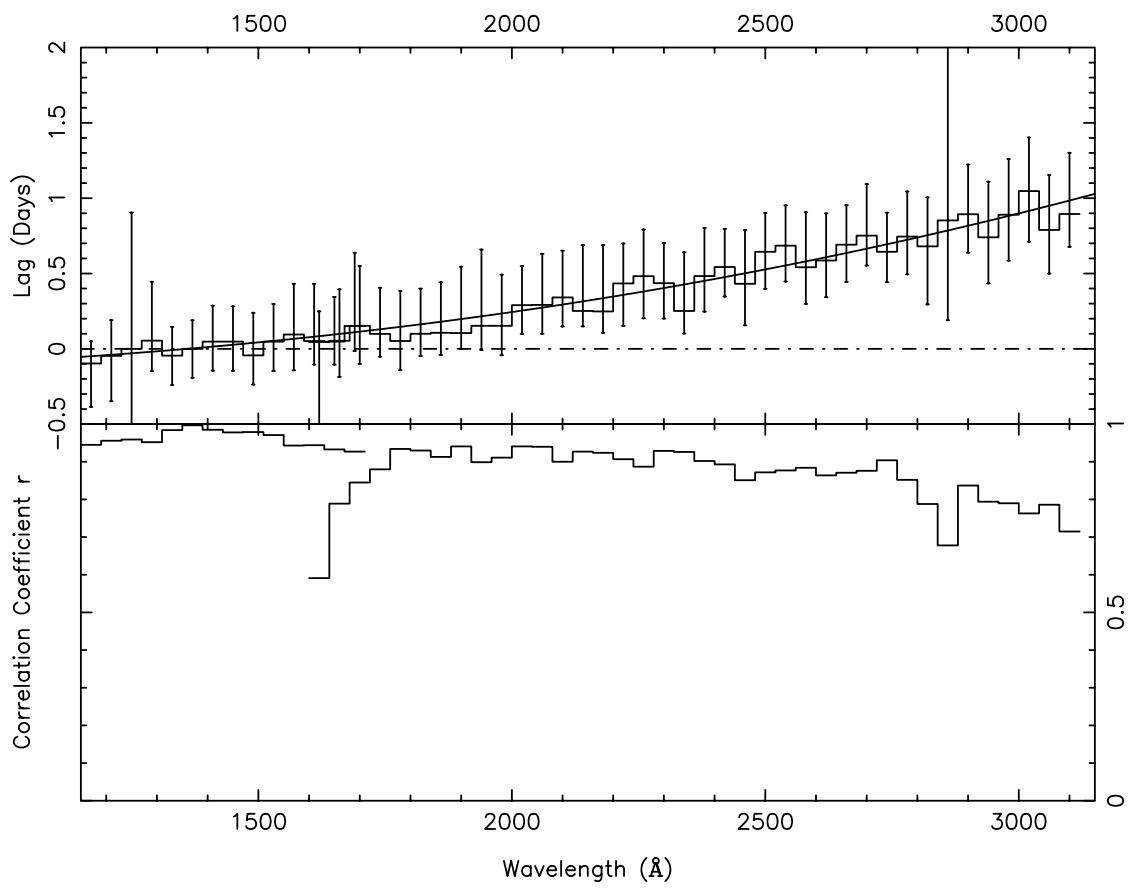

FIG. 10.- UV lag spectrum binned to $40 \AA$ A. Top: The solid histogram denotes the centroid lags. The error bars are those derived from a modelindependent quasi-bootstrap and flux randomization method. The solid line represents the best-fit function $\tau \propto\left(\lambda^{\gamma}-\lambda_{0}^{\gamma}\right)$, with $\lambda_{0}=1365$ and $\gamma=2.4 \pm 0.1$. There is a clear trend of increasing lag with wavelength. Bottom: The solid histogram denotes the maximum correlation coefficient. The $1150-3140 \AA$ variations are well correlated with $r_{\max } \approx 0.9$. The dip in the maximum correlation coefficient at about $1700 \AA \AA$ is due to calibration uncertainties in the G230L spectra. (See text for further details.)

$\gamma=2.4 \pm 0.1$. By including the optical data, the lagwavelength relationship appears to flatten.

\section{EMISSION-LINE VARIABILITY}

The largely featureless rms spectra of Figures 1 and 2 immediately show that any emission-line variability is of low amplitude. Figure 1 shows Ly $\alpha \lambda 1216, \mathrm{~N} v \lambda 1240, \mathrm{Si}$ IV $+\mathrm{O}$ IV $] \lambda 1400, \mathrm{C}$ IV $\lambda 1549$, and $\mathrm{He}$ II $\lambda 1640$ variations are present with amplitudes of less than $4 \%, 5 \%, 6 \%, 5 \%$, and $4 \%$, respectively. Figure 2 indicates $\mathrm{Si}$ III $\lambda 1892, \mathrm{C}$ III $] \lambda 1909$, and $\mathrm{Mg}$ II $\lambda 2798$ variations with amplitudes of less than $6 \%$, $6 \%$, and $4 \%$, respectively. For cases where emission-line

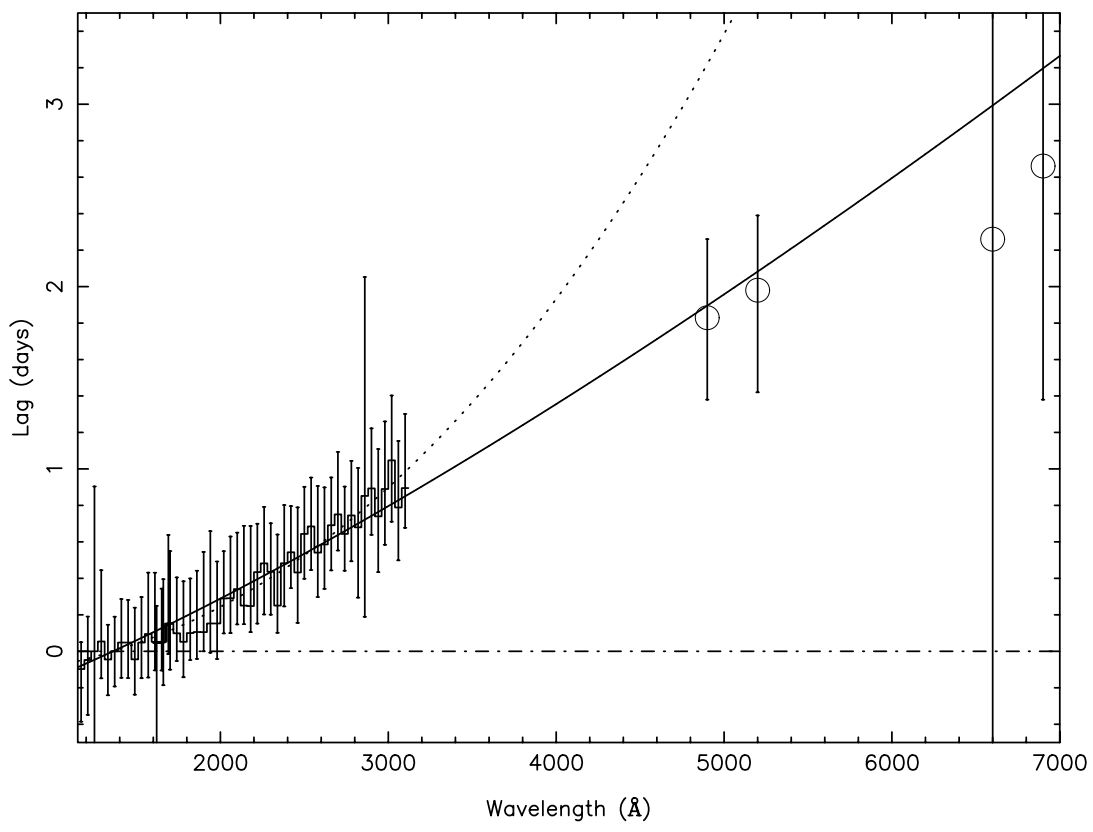

FIG. 11. - UV/optical lag spectrum. The optical lag measurements (open circles) are derived from contemporaneous optical monitoring data presented in Paper III. The solid line represent represents the best-fit function $\tau \propto\left(\lambda^{\gamma}-\lambda_{0}^{\gamma}\right)$, with $\lambda_{0}=1365$ and $\gamma=1.3 \pm 0.1$. The dotted line represents the best-fit function to the UV data, denoted by the solid histogram (detailed in Fig. 10); i.e., $\gamma=2.4 \pm 0.1$. By including the optical data, the lag-wavelength relationship flattens. 
variations are present or suggested, the bulk of the variation occurs in the core of the line. The variations at these wavelengths for emission lines contaminated by intrinsic absorption may be a superposition of both emission- and absorption-line variability. A discussion of the latter is deferred to a later paper. We note the $\mathrm{N} v \lambda 1240$ variations appears to be slightly asymmetric, with a deficit of response in its blue wing. The variations at about $1215 \AA$ are spurious and attributable to time-dependent residuals from imperfect subtraction of geocoronal Ly $\alpha$ emission.

Emission-line light curves were constructed by direct integration, summing the flux in specified, absorption-free wavelength regions after subtracting a continuum defined by a linear fit to the nearest continuum regions on either side of the emission-line region. Our attempts at validating and possibly improving our direct integration light curves through spectral fitting with the IRAF task SPECFIT (Kriss 1994) failed. This was because the spectral complexity of the emission-line profiles permitted too much freedom in the fitting process such that artificial emission-line variations were introduced by corresponding changes in the adopted absorption profile. The blanket exclusion of any absorption regions from our variable emission-line flux regions, defined by their respective rms spectra (Figs. 1 and 2 ), severely inhibited our ability to extract the already low amplitude variations. We were only able to extract significant emission-line variations for $\mathrm{Ly} \alpha \lambda 1216$. The comparatively smaller fluxes and larger measurement errors for the other emission lines precluding extraction of significant variations.

Figure 12 presents the $\operatorname{Ly} \alpha \lambda 1216$ light curves for the monitoring period. The data points with error bars describe the emission-line variations between $1240-1243 \AA$ and $1247-1250 \AA$ ("light curve 1 ”) and the dashed line those between 1240 and $1250 \AA$ ("light curve 2 "); the latter includes any possible variable contribution from the intrinsic $\mathrm{H} \mathrm{I}$ absorption feature. In both cases the continuum is defined by a linear fit between $1155-1180 \AA$ and $1350-1380$ $\AA$. The $1365 \AA$ A light curve (solid line; see $\S 4.1$ ) is scaled and vertically shifted to fit light curve 1 . We note our observed emission-line flux measurements are not corrected for reddening.

The Ly $\alpha \lambda 1216$ variations resemble those of the continuum but with greatly reduced amplitude and appear delayed by about a few days. In each case, the flux increases by $\sim 3 \%$ during the first 15 days, and similar flare events occur at similar times (i.e., JD $-2,450,000 \approx 1697$ and 1711). The contrast of any features in the light curves is poor on account of the low-amplitude variability, which is comparable to the measurement uncertainties. On timescales of about 60 days, the intrinsic line variations are $\sim 1 \%$, with the full range of variation $\sim 7 \%$. Moreover, the Ly $\alpha \lambda 1216$ light curves are clearly well correlated and exhibit similar variability patterns. These results suggest any intrinsic $\mathrm{H}_{\mathrm{I}}$ absorption line variability does not notably affect the emission-line variations.

Cross-correlation of the $\operatorname{Ly} \alpha \lambda 1216$ and $1365 \AA$ variations quantifies their apparent similarity. Our results are presented in Figure 13 and Table 3. Figure 13 presents Ly $\alpha \lambda 1216$ $\mathrm{CCFs}$ for both line light curves detailed above. The solid line and filled data points represent the ICCF and ZDCF CCFs, respectively, as described earlier, for light curve 1, and the dashed line and open data points are for light curve 2. The Ly $\alpha \lambda 1216$ variations are correlated with the $1365 \AA$ variations with a maximum cross-correlation amplitude $r_{\max } \approx 0.5$. The probability of exceeding $r \approx 0.5$ in a random sample of observations (of the same number, $N=45$, as here) drawn from an uncorrelated parent population is about $0.1 \%$ (Bevington \& Robinson 1992, p. 256); therefore, the $\operatorname{Ly} \alpha \lambda 1216$ variations are correlated with those at 1365

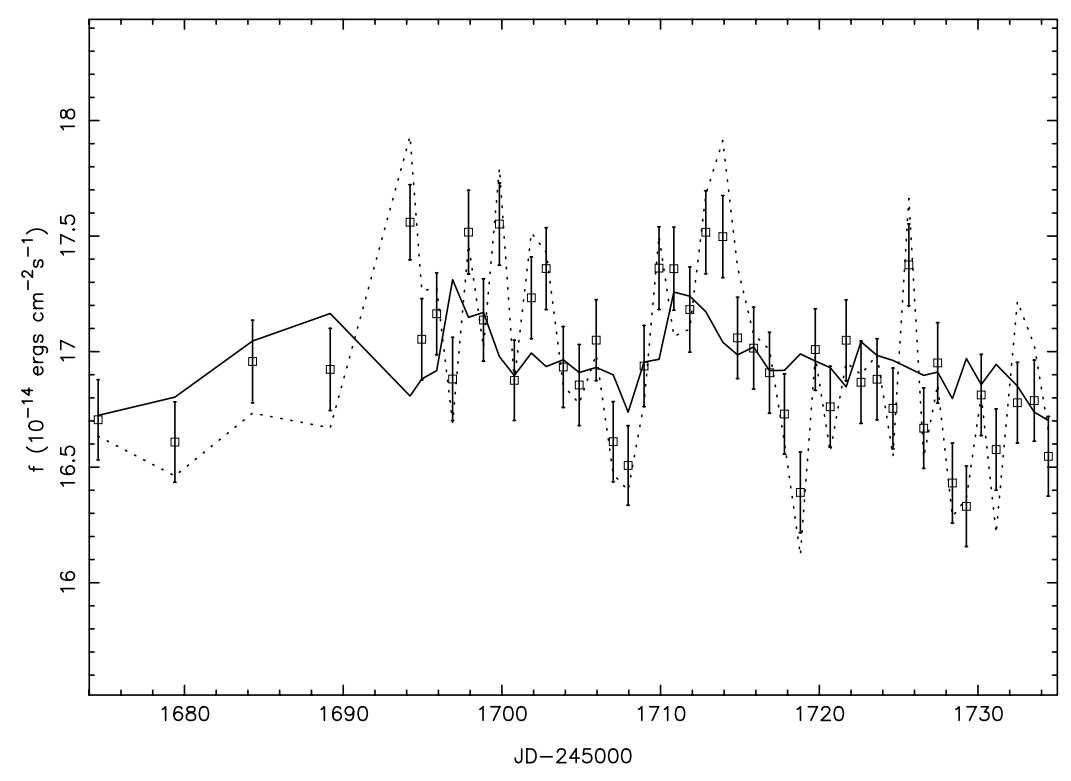

FIG. 12. - Ly $\alpha \lambda 1216$ light curves for the 60 day monitoring period. The data points with error bars describe the emission-line variations between $1240-1243 \AA$ and $1247-1250 \AA$ and the dashed line those between 1240 and $1250 \AA$. In both cases the continuum is defined by a linear fit between $1155-1180$ $\AA$ and $1350-1380 \AA$. The $1365 \AA$ light curve $(\S 4.1$; Fig. 6) is scaled and vertically shifted to fit light curve 1 and is shown by the solid line. The emission-line variations denoted by the dashed line nclude any possible variable contribution from the intrinsic $\mathrm{H}$ I absorption feature. The intrinsic Ly $\alpha \lambda 1216$ flux amplitude variations are about $1 \%$, and both light curves exhibit similar qualitative behavior, which in turn mimics those of the continuum regions. We note the dashed line variations have been normalized to have the same mean value as those denoted by the data points. 


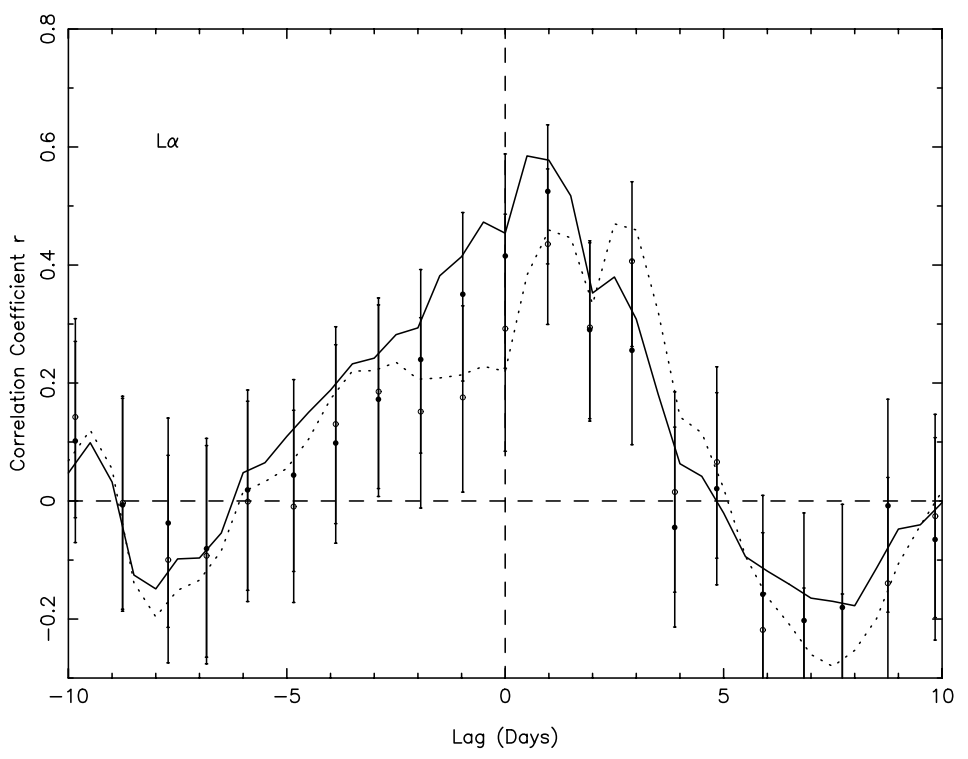

FIG. 13. - CCFs for the Ly $\alpha \lambda 1216$ emission ine. The solid line and filled data points represent the ICCF and ZDCF CCFs, respectively, for light curve 1 , and the dashed line and open data points are for light curve 2 (see Fig. 12). The Ly $\alpha \lambda 1216$ variations are correlated with the $1365 \AA$ variations with a maximum cross-correlation amplitude $r_{\max } \approx 0.5$. Their CCFs peak away from zero at about 2 days, and the differences between them are not statistically significant.

$\AA$ at about $99.9 \%$ confidence. The formal significance of this correlation is most likely overestimated, since not all correlated data points are independent. Detailed simulations will be required in order to quantify the magnitude of this bias. By directly cross-correlating light curves 1 and 2, we confirm they are correlated with essentially zero time delay. The peak and centroid lag measurements for light curves 1 and 2 are consistent to within the large uncertainties. Moreover, for light curve 1 the lag measurements are consistent with zero delay. Our results only permit an upper limit for the size of the Ly $\alpha \lambda 1216$ emission region, $R_{\mathrm{BLR}}^{\mathrm{Ly} \alpha} \lesssim 3$ 1t-days. We caution that the low-amplitude $\operatorname{Ly} \alpha \lambda 1216$ variations may indicate the bulk of the emission region is at larger radii, as discussed in $\S 6.2$.

\section{DISCUSSION}

\subsection{Nature of the UV Broad Line Region}

A complete study of the nature of the UV BLR of Ark 564 requires detailed photoionization modeling of the spectral energy distribution and is beyond the scope of this paper. Here we offer a preliminary discussion of our results in the context of previous work.

The BLR of Ark 564 is characterized by smaller gas velocity dispersions (as determined by the emission-line velocity FWHM) than found in typical S1s (see $\S 3$ ), as noted earlier by Rodríguez-Pascual et al. (1997a), i.e., about $2000 \mathrm{~km} \mathrm{~s}^{-1}$ compared to $5000 \mathrm{~km} \mathrm{~s}^{-1}$. These authors found evidence for similar magnitude UV line widths in about a dozen NLS1s (see also Kuraszkiewicz et al. 2000). The full widths of the low- and high-ionization lines are comparable (see Fig. 4) and interestingly similar to those of the $\mathrm{N}$ III] $\lambda 1750$ and $\mathrm{C}$ III] $\lambda 1909$ intercombination lines. For example, the full widths of He II $\lambda 1640, N$ III] $\lambda 1750$, C III] $\lambda 1909$, and $\mathrm{Mg}$ II $\lambda 2798$ are $1800,1900,1900$, and $1700 \mathrm{~km} \mathrm{~s}^{-1}$, respectively (as measured from the G230L spectra; Table 1).

As noted in $\S 3$, the broad emission-line profiles of Ark 564 are approximately symmetric about their systemic wavelengths. These results, and those above, contrast with UV/optical observations of quasars (e.g., Gaskell 1982; Wilkes 1984; Corbin 1991) and S1s, showing that highionization lines are shifted blueward of low-ionization lines, which are approximately at systemic redshifts and slightly broader. Leighly (2000) presented observations of two NLS1s (IRAS 13224-3809 and 1H 0707-495), which also showed the high-ionization lines were strongly blueshifted and much broader than the low-ionization Mg II $\lambda 2798$ line, similar to results for the NLS1s I Zw 1 and NGC 4051 presented by Laor et al. (1997) and Peterson et al. (2000), respectively. Collinge et al. (2001) find the $\mathrm{C}$ IV $\lambda 1549$ line in NGC 4051 to be blueshifted by about $100 \mathrm{~km} \mathrm{~s}^{-1}$. These prior results are often interpreted as evidence for BLR radial motions. The low-ionization lines may arise in an accretion disk, with the high-ionization lines emitted in a wind launched from this disk (e.g., Collin-Souffrin et al. 1988). Our results suggest an absence of outflowing highionization BLR gas with velocities $\gtrsim 230 \mathrm{~km} \mathrm{~s}^{-1}$ in Ark 564. Moreover, the approximate symmetry of the emissionline profiles may indicate the predominance of optically thin broad-line emission, since it is emitted essentially isotropically and traces the full velocity field (Netzer 1976; Davidson 1977; Shields, Ferland, \& Peterson 1995).

The level of intrinsic line variability is notably less than observed in typical S1s over comparable timescales (§ 5). For example, NGC 5548 (Korista et al. 1995) showed Ly $\alpha$ $\lambda 1216, \mathrm{C}$ IV $\lambda 1549$, and $\mathrm{He}$ II $\lambda 1640$ variations of $\sim 13 \%$, $\sim 9 \%$, and $\sim 14 \%$, respectively. Similarly, the same lines in NGC 7469 (Wanders et al. 1997) showed variations of $\sim 10 \%, \sim 9 \%, \sim 14 \%$. This difference may simply be a consequence of the reduced level of assumed ionizing continuum variations combined with geometrical dilution owing to the finite BLR size. To investigate this scenario we convolved the observed continuum variations with thin-shell BLRs at radii of 6 and 2 1t-days (i.e., the predicted sizes of the Ly $\alpha \lambda 1216$ and He II $\lambda 1640$ emission regions; see $\S 6.2$ ), respectively. In these cases, we find $\mathrm{Ly} \alpha \lambda 1216$ and $\mathrm{He}$ II $\lambda 1640$ variations on the order of $3 \%$ and $6 \%$, respectively. 


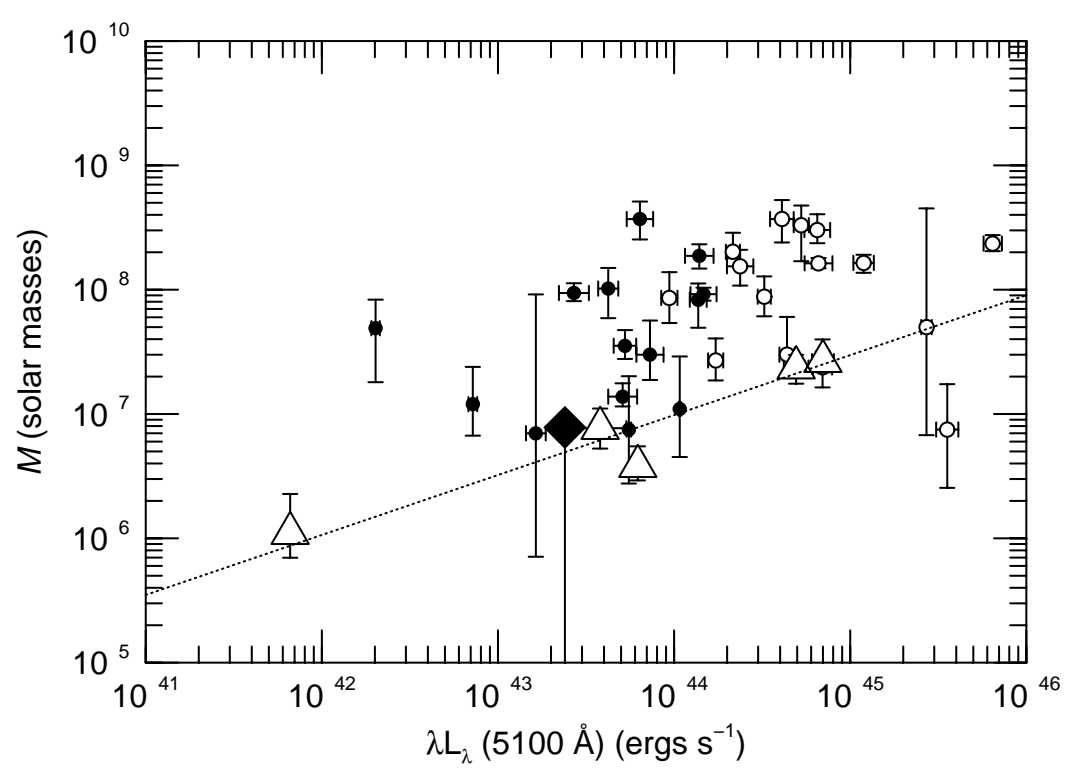

FIG. 14.- Reverberation-based virial mass and optical luminosity relationship for AGNs. The filled circles are Seyfert galaxies from Wandel et al. (1999), and the open circles are QSOs from Kaspi et al. (2000). The large triangles are the AGNs from the same sources whose broad lines have widths less than $\sim 2000 \mathrm{~km} \mathrm{~s}^{-1}$. Our upper limit mass estimate for Ark 564 is shown as a filled diamond. The best-fit regression line based on the narrow-line objects is denoted by the dashed line, with $M \propto L^{0.48 \pm 0.08}$.

These results suggest that this type of scenario does not completely explain the reduced level of emission-line variability in Ark 564. On the other hand, the line-emitting gas in Ark 564 may be fully ionized in hydrogen and, hence, optically thin to the Lyman continuum. This would lead to greatly reduced emission-line variability, with detectable variations occurring only for sufficiently large continuum variations that induce significant changes in the ionization structure of the emission region. Similarly, this scenario alone is likely insufficient to account for the lack of line variability across the observed range of ionization, as evidenced by the calculations of Ferland \& Mushotzky (1982). However, we note these calculations are more appropriate for S1 BLRs and therefore may be misrepresentative of NLS1s. Some combination of both the above effects appears a reasonable explanation for the reduced level of emission-line variability in Ark 564.

The unusual UV spectral properties of Ark 564 (§ 3), combined with stronger ionizing fluxes relative to other S1s, suggest NLS1 BLRs have a physically distinct nature from those found in S1s and quasars. Kuraszkiewicz et al. (2000) posit NLS1 BLRs characterized by gas cloud densities $n_{\mathrm{H}} \approx 10^{11} \mathrm{~cm}^{-3}$, ionization parameters $U \approx 0.01$, and BLR radii $R_{\mathrm{BLR}}^{\mathrm{UV}} \approx 20$ lt-days. On the other hand, RodríguezPascual et al. $(1997 \mathrm{a})$ posit $n_{\mathrm{H}} \approx 10^{7.5} \mathrm{~cm}^{-3}, U \approx 0.3$, and BLR radii $R_{\mathrm{BLR}}^{\mathrm{UV}} \approx 2$ lt-days; under these conditions the broad-line-emitting gas becomes fully ionized in hydrogen and thereby optically thin to the Lyman continuum. Our emission-line profiles and variability results hint at a predominance of optically thin broad-line-emitting gas in Ark 564. In combination with a small estimated BLR size (see $\S 5)$ of $\lesssim 3$ 1t-days, these conditions appear more readily reconcilable with those suggested by Rodríguez-Pascual et al. (1997a), as noted above.

\subsection{Evidence for a Black Hole Accretion Disk System}

The wavelength-dependent continuum time delays of $\S 4.2$ suggest a stratified continuum reprocessing region extending a couple of light days from the central black hole and provide possible evidence for an accretion disk structure (Collier et al. 1998). Simple irradiated accretion disk models with radial temperature profiles $T \propto R^{-3 / 4}$ predict $\tau \propto \lambda^{4 / 3}$. Flatter temperature profiles predict steeper $\tau-\lambda$ relationships and are realizable with, e.g., irradiated flared accretion disk models. These models may be able to explain the steeper UV lag spectrum presented in $\S$ 4.2. The fine details are determined by the geometry and structure of the accretion disk, relative prominence of viscous heat dissipation and irradiation effects, and the emission physics. Vaughan et al. (1999) present evidence, based on X-ray data, for a strongly ionized disk in Ark 564. Given the close proximity of the UV- and X-ray-emitting regions ( $\$ 4$; Papers I and III), our results support their conclusions. In the case of NGC 7469 , the only other source with a clear detection of wavelength-dependent continuum lags (Wanders et al. 1997; Collier et al. 1998; Kriss et al. 2000), the UV/optical lag spectrum was consistent with $\tau \propto \lambda^{4 / 3}$ and is similar to that observed in $\S 4$ 4.2. On the other hand, these continuum lags may be a result of diffuse continuum emission from broad-line-emitting clouds (Korista \& Goad 2000) or some combination of both these effects.

In $\S 5$ we estimated an upper limit of 31 t-days for the size of the Ly $\alpha \lambda 1216$ broad-line-emitting region, $R_{\mathrm{BLR}}^{\mathrm{Ly} \alpha}$. We may compare this estimate with that expected on the basis of results obtained for other AGNs by using the Balmer emission region size $R_{\mathrm{BLR}}^{\mathrm{H} \beta}$-luminosity relationships of Kaspi et al. (2000) and Peterson et al. (2000). The luminosity $\lambda L_{\lambda}(5100 \AA)=\lambda F_{\lambda}(5100 \AA) 4 \pi D^{2} \approx 2.4 \times 10^{43} \mathrm{ergs} \mathrm{s}^{-1}$, adopting a mean flux $F_{\lambda}(5100 \AA)=4 \times 10^{-15} \mathrm{ergs} \mathrm{s}^{-1}$ $\mathrm{cm}^{-2} \AA^{-1}$ and a distance $D=c z / H_{0}=99 \mathrm{Mpc}$ (assuming $H_{0}=75 \mathrm{~km} \mathrm{~s}^{-1} \mathrm{Mpc}^{-1}$ ). This leads to an estimate for the size of the $\mathrm{H} \beta$-emitting region $R_{\mathrm{BLR}}^{\mathrm{H} \beta} \approx 12$ lt-days from the $R_{\mathrm{BLR}}^{\mathrm{H} \beta}$-luminosity relationship shown in Figure 6 of Peterson et al. (2000). From previous monitoring programs on S1s (Netzer \& Peterson 1997), we can estimate the size of the Ly $\alpha \lambda 1216$-emitting region to be $R_{\mathrm{BLR}}^{\mathrm{Ly} \alpha} \approx 0.5 R_{\mathrm{BLR}}^{\mathrm{H} \beta}$. Similarly, 
we expect that the highest ionization lines, $\mathrm{N} v \lambda 1240$ and He II 21640 , arise in a region of extent $\sim 0.2 R_{\mathrm{BLR}}^{\mathrm{H} \beta}$. On the basis of the optical luminosity of Ark 564, we thus predict $R_{\mathrm{BLR}}^{\mathrm{Ly} \alpha} \approx 6$ lt-days and $R_{\mathrm{BLR}}^{\mathrm{Nv}} \approx 2$ 1t-days. The factor of $\sim 2$ difference between our measured and predicted values for $R_{\mathrm{BLR}}^{\mathrm{Ly} \alpha}$ is probably not significant, since the intrinsic scatter in the $R_{\mathrm{BLR}}^{\mathrm{H} \beta}$-luminosity relationship is on the order of a factor of 5. These results give us confidence that our $R_{\mathrm{BLR}}^{\mathrm{Ly} \alpha}$ estimate is in the right ballpark.

By combining our $R_{\mathrm{BLR}}^{\mathrm{Ly} \alpha}$ upper limit of 31 t-days with the emission-line velocity FWHM $V_{\mathrm{FWHM}} \approx 2500 \mathrm{~km} \mathrm{~s}^{-1}$ derived from the rms emission-line profile (and assumed to be gravitationally determined), we estimate a virial mass upper limit from

$$
M=f V_{\mathrm{FWHM}}^{2} R_{\mathrm{BLR}}^{\mathrm{Ly} \alpha} / G \lesssim 8 \times 10^{6} M_{\odot},
$$

where for consistency with Wandel et al. (1999) and Kaspi et al. (2000) we use $f=3 / \sqrt{2}$. This mass estimate is consistent with the independent mass estimate of $M \sim 1 \times 10^{7}$ $M_{\odot}$ of Pounds et al. (2001) based on a fluctuation power spectrum analysis of X-ray variability in Ark 564. And it is consistent with our mass estimate based on the variability arguments of $\S 4.1$. Furthermore, we note that the position of Ark 564 in the AGN mass-luminosity plane defined by Figure 7 of Peterson et al. (2000) is consistent with the bestfit regression line on the basis of the NLS1s alone (see Fig. 14). This is consistent with the hypothesis that Ark 564 harbors a comparatively small black hole accreting with a higher accretion rate and/or is viewed more face-on than typical S1s,. as found earlier by Pounds et al. (2001).

We conclude with a cautionary note concerning our derived upper limits for the size of Ark 564's Ly $\alpha \lambda 1216$ BLR and black hole mass. The reduced continuum to Ly $\alpha$ $\lambda 1216$ variations ratio of $\sim 4$, relative to $\sim 1$ for the S1s NGC 5548 and NGC 7469 (references as given in $\S 6.1$ ), suggests our $\mathrm{Ly} \alpha \lambda 1216$ reverberations arise from a region only about $\frac{1}{4}$ of the size of the Ly $\alpha \lambda 1216$ region probed by reverberation in S1s and is therefore biased low. To illustrate the potential importance of this bias, we assume that the $\operatorname{Ly} \alpha \lambda 1216$ BLR is a thin shell of radius $R$. Equal time delay intervals define equal areas on the shell, and if our Ly $\alpha \lambda 1216$ response from less than 31 t-days is from only $\frac{1}{4}$ of the area of the shell, we underestimate $R$ by a factor of 4 . This would lead to an $R_{\mathrm{BLR}}^{\mathrm{Ly} \alpha}$ upper limit of 12 1t-days. The bias in our black hole mass upper limit depends on the unknown nature of the gas velocity field and is not readily quantifiable without better data.

\section{SUMMARY}

An intensive 2 month monitoring program on the NLS1 Ark 564 was undertaken with $H S T$ during 2000 May 9-July 8 . We summarize our results as follows.
1. The fractional variability amplitude of the UV continuum variations between 1365 and $3000 \AA$ is about $6 \%$ on timescales of 60 days. This level of variability is about a factor of 3 less than that found in S1s. We find evidence for fast, large-amplitude continuum variations (e.g., trough-topeak flux changes of $\sim 18 \%$ in about 3 days) atypical of those found in S1s that display similar amplitude variations on longer timescales.

2. We present evidence for wavelength-dependent continuum time delays. The continuum variations at $3000 \AA$ lag behind those at $1365 \AA$ by about 1 day. By combining the UV data with the optical data described in Paper III, we find that the variations at $5200 \AA$ lag behind those at $1365 \AA$ by about 2 days. These delays may be interpreted as evidence for a stratified continuum reprocessing region, possibly an accretion disk structure. However, the delays may be a result of diffuse continuum emission from broad-lineemitting clouds or some combination of both these effects.

3. The $\operatorname{Ly} \alpha \lambda 1216$ emission line exhibits intrinsic rms variations of about $1 \%$ on timescales of 60 days. These variations lag those at $1365 \AA$ by $\lesssim 3$ days, and combining this with the line width yields a putative black hole mass limit of $\lesssim 8 \times 10^{6} M_{\odot}$. This calculation assumes the line width is determined by the gravity of the black hole. We caution that the low-amplitude Ly $\alpha \lambda 1216$ variations may indicate the bulk of the emission region is at larger radii; therefore, the veracity of our black hole mass limit is questionable. The possible bias in our black hole mass estimate is uncertain given the unknown nature of the gas velocity field. Despite the unreliability of our mass estimate, it is consistent with the independent estimate $M \sim 1 \times 10^{7} M_{\odot}$ of Pounds et al. (2001), based on a fluctuation power spectrum analysis of X-ray variability in Ark 564.

4. The rms spectra suggest other strong emission-line variability (e.g., of C IV $\lambda 1549$ and $\mathrm{He}$ II $\lambda 1640$ ) occurs with rms amplitudes of less than 5\%. The low level of NLS1 emission-line variability is in contrast to that found in typical S1s, which display rms flux variations of $\sim 10 \%$ on similar timescales.

We are pleased to acknowledge support for this work by NASA through grant HST-GO-08265.01-A from the Space Telescope Science Institute, which is operated by the Association of Universities for Research in Astronomy, Inc., under NASA contract NAS 5-26555. This research has made use of the NASA/IPAC Extragalactic Database (NED), which is operated by the Jet Propulsion Laboratory, California Institute of Technology, under contract with the National Aeronautics and Space Administration. SM acknowledges NASA grant NAG 5-8913 (LTSA). We are grateful to an anonymous referee for useful comments.

\section{REFERENCES}

Alexander, T. 1997, in Astronomical Time Series, ed. D. Maoz, A. Sternberg, \& E. M. Leibowitz (Dordrecht: Kluwer), 163

Bevington, P., \& Robinson, D. 1992, Data Reduction and Error Analysis for the Physical Sciences (New York: McGraw-Hill)

Blandford, R., \& McKee, C. F. 1982, ApJ, 255, 419

Boller, T., Brandt, W. N., \& Fink, H. 1996, A\&A, 305, 53

Boroson, T. A., \& Green, R. F. 1992, ApJS, 80, 109

Brandt, W. N., Mathur, S., \& Elvis, M. 1997, MNRAS, 285, L25

Clavel, J., et al. 1991, ApJ, 366, 64

Collier, S., et al. 1998, ApJ, 500, 162

Collier, S., Horne, K., Wanders, I., \& Peterson, B. M. 1999, MNRAS, 302,

L24
Collier, S., Peterson, B. M., \& Horne, K. 2001, in ASP Conf. Ser. 224 Probing the Physics of Active Galactic Nuclei by Multiwavelength Monitoring, ed. B. M. Peterson, R. S. Polidan, \& R. W. Pogge (San Francisco: ASP), 457

Collinge, J., et al. 2001, ApJ, 557, 2

Collin-Souffrin, S., Dyson, J., McDowell, J., \& Perry, J. 1988, MNRAS, 232, 539

Corbin, M. 1991, ApJ, 371, L51

Crenshaw, M., Kraemer, S., Boggess, A., Maran, S., Mushotzky, R., \& Wu, C. 1999, ApJ, 516, 750

Crenshaw, D. M., et al. 2001, ApJ, submitted

Davidson, K. 1977, ApJ, 218, 20 
Edelson, R., Krolik, J., \& Pike, G. 1990, ApJ, 359, 86

Edelson, R., Turner, T. J., Pounds, K. A., Vaughn, S., Markowitz, A., Marshall, H., Dobbie, P., \& Warwick, R. 2001, ApJ, in press

Ferland, G., \& Mushotzky, R. F. 1982, ApJ, 262, 564

Gaskell, C. M. 1982, ApJ, 263, 79

Gaskell, C. M., \& Sparke, L. 1986, ApJ, 305, 175

Giannuzzo, M. E., Mignoli, M., Stirpe, G., \& Comastri, A. 1998, A\&A, 330, 894

Guilbert, P. W., Fabian, A. C., \& McCray, R. 1983, ApJ, 266, 466

Huchra, J. P., Vogeley, M. S., \& Geller, M. J. 1999, ApJS, 121, 287

Kaspi, S., Smith, P. S., Netzer, H., Maoz, D., Jannuzi, B. T., \& Giveon, U. 2000, ApJ, 533, 631

Korista, K., et al. 1995, ApJS, 97, 285

Korista, K., \& Goad, M. 2000, ApJ, 536, 284

Kriss, G. 1994, in ASP Conf. Ser. 61, Astronomical Data Analysis Software and Systems III, ed. D. Crabtree, R. J. Hanisch, \& J. Barnes (San Francisco: ASP), 437

Kriss, G., Peterson, B. M., Crenshaw, D. M., \& Zheng, W. 2000, ApJ, 535, 58

Kuraszkiewicz, J., Wilkes, B., Czerny, B., \& Mathur, S. 2000, ApJ, 542, 692

Laor, A., Fiore, F., Elvis, M., Wilkes, B., \& McDowell, J. C. 1997, ApJ, 477, 93

Leighly, K. M. 1999a, ApJS, 125, 297

1999b, ApJS, 125, 317

2000, NewA Rev., 44, 395

Lindler, D. 1998, CALSTIS Reference Guide (CALSTIS Version 5.1)

Madau, P. 1988, ApJ, 327, 116

Mathur, S., Elvis, M., \& Wilkes, B. 1995, ApJ, 452, 230

Nandra, K., George, I. M., Mushotzky, R. F., Turner, T. J., \& Yaqoob, T. 1997, ApJ, 477, 602

Netzer, H. 1976, MNRAS, 177, 473

Netzer, H., \& Peterson, B. M. 1997, in Astronomical Time Series, ed. D. Maoz, A. Sternberg, \& E. M. Leibowitz (Dordrecht: Kluwer), 85

O'Brien, P., Wilson, R., \& Gondhalekar, P. M. 1988, MNRAS, 233, 801

Osterbrock, D. E., \& Pogge, R. W. 1985, ApJ, 297, 166

Peterson, B. M. 2001, in Advanced Lectures on the Starburst-AGN Connection, ed. I. Aretxaga, D. Kunth, \& R.Mújica (London: World Scientific), 3
Peterson, B. M., et al. 2000, ApJ, 542, 161

Peterson, B. M., Wanders, I., Horne, K., Collier, S., Alexander, T., Kaspi, S., \& Maoz, D. 1998, PASP, 110, 660

Pounds, K., Done, C., \& Osborne, J. 1995, MNRAS, 277, L5

Pounds, K., Edelson, R., Markowitz, A., \& Vaughan, S. 2001, ApJ, 550, L15

Puchnarewicz, E. M., Mason, K., Córdova, F., Kartje, J., BranduardiRaymont, G., Mittaz, J., Murdin, P., \& Allington-Smith, J. 1992, MNRAS, 256, 589

Rodríguez-Pascual, P. M., Mas-Hesse, J. M., \& Santos-Lleó, M. 1997a, A\&A, 327, 72

Rodríguez-Pascual, P. M., et al. 1997b, ApJS, 110, 9

Rokaki, E., \& Boisson, C. 1999, MNRAS, 307, 41

Schlegel, D. J., Finkbeiner, D. P., \& Davis, M. 1998, ApJ, 500, 525

Shemmer, O., et al. 2001, ApJ, 561 (Paper III)

Shields, J. C., Ferland, G. J., \& Peterson, B. M. 1995, ApJ, 441, 507

Turner, J., et al. 1999, in Proc. 19th Texas Symp. on Relativistic Astrophysics \& Cosmology, ed J. Paul, T. Montmerle, \& E. Aubourg (Gif-surYvette: CEA-Saclay), E441

Turner, J., et al. 2001, ApJ, 561 (Paper I)

van Groningen, E. 1993, A\&A, 272, 25

Vaughan, S., Pounds, K., Reeves, J., Warwick, R., \& Edelson, R. 1999, MNRAS, 308, L34

Walter, R., \& Fink, H. 1993, A\&A, 274, 105

Wandel, A., \& Boller, T. 1998, A\&A, 331, 884

Wandel, A., Peterson, B. M., \& Malkan, M. A. 1999, ApJ, 526, 579

Wanders, I., et al. 1997, ApJS, 113, 69

Welsh, W., \& Horne, K. 1991, ApJ, 379, 586

Welsh, W. F., Peterson, B. M., Koratkar, A. P., \& Korista, K. T. 1998, ApJ, 509,118

White, R. J., \& Peterson, B. M. 1994, PASP, 106, 879

Wilkes, B. 1984, MNRAS, 207, 73

Wilkes, B., Kuraszkiewicz, J., Green, P., Mathur, S., \& McDowell, J. C. 1999, ApJ, 513, 76 